\title{
Strain Hardening of Polymer Glasses: Entanglements, Energetics, and Plasticity
}

\author{
Robert S. Hoy ${ }^{*}$ and Mark O. Robbins \\ Department of Physics and Astronomy, Johns Hopkins University, Baltimore, MD 21218
}

(Dated: January 5, 2008)

\begin{abstract}
Simulations are used to examine the microscopic origins of strain hardening in polymer glasses. While stress-strain curves for a wide range of temperature can be fit to the functional form predicted by entropic network models, many other results are fundamentally inconsistent with the physical picture underlying these models. Stresses are too large to be entropic and have the wrong trend with temperature. The most dramatic hardening at large strains reflects increases in energy as chains are pulled taut between entanglements rather than a change in entropy. A weak entropic stress is only observed in shape recovery of deformed samples when heated above the glass transition. While short chains do not form an entangled network, they exhibit partial shape recovery, orientation, and strain hardening. Stresses for all chain lengths collapse when plotted against a microscopic measure of chain stretching rather than the macroscopic stretch. The thermal contribution to the stress is directly proportional to the rate of plasticity as measured by breaking and reforming of interchain bonds. These observations suggest that the correct microscopic theory of strain hardening should be based on glassy state physics rather than rubber elasticity.
\end{abstract}

PACS numbers: 61.41.+e,81.05.Kf,81.40.Jj,81.40.Lm

\section{INTRODUCTION}

Glass forming polymers are of great industrial importance and scientific interest because of their unique mechanical properties, which arise from the connectivity and random-walk-like structure of the constituent chains. At large strains, the stress increases as the chain molecules orient, in a process known as strain hardening. Strain hardening suppresses strain localization (crazing, necking, shear banding) and is critical in determining material properties such as toughness and wear resistance.

Traditional theories of glassy strain hardening [1, 2] assume that polymer glasses behave like crosslinked rubber, with the number of monomers between crosslinks equal to the entanglement length $N_{e}$. The increase in the stress $\sigma$ due to deformation by a stretch tensor $\bar{\lambda}$ is attributed to the decrease in entropy as polymers stretch affinely between entanglements. Beyond the initial plastic flow regime, entropic network models predict [2]:

$$
\sigma(\bar{\lambda})=\tau_{\text {flow }}+G_{R} g(\bar{\lambda}) L^{-1}(h) / 3 h
$$

where $\tau_{\text {flow }}$ is the plastic flow stress, $G_{R}$ is the strain hardening modulus, $L^{-1}$ is the inverse Langevin function, $g(\bar{\lambda})$ describes the entropy reduction for ideal Gaussian chains, and $L^{-1}(h) / 3 h$ corrects for the finite length of segments between entanglements. The value of $N_{e}$ enters in $h$, which is the ratio of the Euclidean distance between entanglements to the contour length.

Entropic network models have had much success in fitting experimental data [2, [3, 4, ,5]. However, serious discrepancies between the models and experiments are revealed in trends with temperature and the values of fit parameters [6]. Entropic models predict $G_{R}=\rho_{e} k_{B} T$,

*Electronic address: robhoy@mrl.ucsb.edu where $\rho_{e}$ is the entanglement density. This is about 100 times smaller than values measured near the glass transition temperature $T_{g}$ [7]. Moreover, $G_{R}$ increases monotonically as $T$ decreases [7, 8], while any entropic stress must drop to zero as $T \rightarrow 0$. Parameters such as $G_{R}$ and $N_{e}$, which entropic models assume to be material constants, must be adjusted significantly to fit data for different strain states (i.e. uniaxial or plane strain) [9]. Fits to the shape of strain hardening curves also yield smaller values of $N_{e}$ than those obtained from the plateau moduli of melts [2].

A more fundamental conceptual difficulty with entropic models is that, unlike rubber, glasses are not ergodic. For $T<T_{g}$, thermal activation is not sufficient to allow chains to sample conformations freely. Rearrangements occur mainly under active deformation 10, 11 and at a frequency that scales with the strain rate [12]. In such far from equilibrium situations, the relevance of entropy is unclear. In addition, experiments [13, 14, 15] and simulations [16, 17, 18] show that the internal energy contributes to strain hardening, but this is not included in entropic network models.

Molecular simulations allow a full analysis of the mechanisms of large strain deformation in glassy polymers $10,18,19,20,21,22,23,24,25,26$. In recent papers 8, 17], we have examined the origins of strain hardening using a coarse-grained bead-spring model 27]. As in experiments, numerical values of $G_{R}$ are much larger than predicted by entropic models and show the opposite trend with temperature [8]. A direct correlation between $\tau_{\text {flow }}$ and $G_{R}$ was discovered that allowed curves for different interactions, strain rates and temperatures to be collapsed [8]. Substantial strain hardening was observed for chains that are shorter than $N_{e}$ and thus do not form a network [17, 25]. For chains of all lengths, strain hardening was directly related to strain-induced orientation and the rate of plastic rearrangement 17 . 
This paper extends our simulation studies in several directions. Uniaxial and plane strain compression are examined for a wide range of $N_{e}, T$ and chain lengths. Stress curves for all entangled systems can be fit to Eq. 1. The fits confirm the connection between $\tau_{\text {flow }}$ and $G_{R}$ [8], which both drop linearly to zero as $T$ rises to $T_{g}$. This observation motivates a modification of Eq. 1. Using $T_{g}$ and the fit to a stress-strain curve at one temperature, the model predicts strain hardening curves for all $T<$ $T_{g}$ remarkably well. However, as in experiments [9], it is necessary to vary parameters such as $G_{R}$ and $N_{e}$ in unphysical ways in order to fit curves for different strain states.

Direct examination of entropic and energetic contributions to strain hardening reveals qualitative failures of network models. The rapid hardening at large strains that is fit by the Langevin correction in Eq. 1 does not reflect a reduction in entropy. Instead there is a rapid rise in energetic stress as chains are pulled taut between entanglements. Variation in the energetic contribution for different strain states leads to changes in fit values of $N_{e}$. For all chain lengths and $N_{e}$, we find that the thermal part of the stress correlates directly with breaking and reformation of van der Waals bonds during deformation. This provides an explanation for the correlation between $G_{R}$ and $\tau_{\text {flow }}$.

Remarkable shape recovery is observed in experiments when highly deformed samples are unloaded and heated slightly above $T_{g}$ [28]. Network models assume this recovery is driven by a "back stress" related to the entropy of the entanglement network, and shape recovery is often seen as providing evidence for entropic strain hardening. Our simulations also show dramatic shape recovery that is driven by orientational entropy. However, the magnitude of the associated stress is only of order $\rho_{e} k_{B} T$ and thus much too small to be significant in strain hardening.

Changes in microscopic chain conformations are also explored. While Eq. 1 assumes affine deformation of segments of length $N_{e}$, the observed deformation becomes increasingly subaffine as strain increases. This reflects a straightening of segments between entanglements that disturbs the local structure of the glass and increases the internal energy. Although unentangled chains do not form a network, they still undergo significant orientation during strain [8, 25]. For all chain lengths, the thermal contribution to the stress is directly related to the orientation of chains on the end-to-end scale rather than the macroscopic stretch [17, 29].

The following section describes the potentials, geometry and strain protocol used in our simulations. Next, fits to entropic network models are examined, and an extension that incorporates the correlation between $\tau_{\text {flow }}$ and $G_{R}$ is presented. This is followed by a discussion of the energetic and entropic contributions to the stress and the role of entropic back stresses in shape recovery. Sections IIID and IIIE explore the effect of chain length and orientation and demonstrate the connection between plastic deformation and the thermal component of the stress. The final section presents a summary and conclusions.

\section{POLYMER MODEL AND METHODS}

We employ a coarse-grained bead-spring polymer model [27] that incorporates key physical features of linear homopolymers such as covalent backbone bonds, excluded-volume and adhesive interactions, chain stiffness, and the topological restriction that chains may not cross. Each linear chain contains $N$ spherical monomers of mass $m$. All monomers interact via the truncated and shifted Lennard-Jones potential:

$$
U_{L J}(r)=4 u_{0}\left[\left(\frac{a}{r}\right)^{12}-\left(\frac{a}{r}\right)^{6}-\left(\frac{a}{r_{c}}\right)^{12}+\left(\frac{a}{r_{c}}\right)^{6}\right]
$$

where $r_{c}$ is the potential cutoff radius and $U_{L J}(r)=0$ for $r>r_{c}$. We express all quantities in terms of the molecular diameter $a$, energy scale $u_{0}$, and characteristic time $\tau_{L J}=\sqrt{m a^{2} / u_{0}}$.

Covalent bonds between adjacent monomers on a chain are modeled using the finitely extensible nonlinear elastic (FENE) potential

$$
U_{F E N E}(r)=-\frac{k R_{0}^{2}}{2} \ln \left(1-\left(\mathrm{r} / \mathrm{R}_{0}\right)^{2}\right),
$$

with the canonical parameter choices [27] $R_{0}=1.5 a$ and $k=30 u_{0} / a^{2}$. The equilibrium bond length $l_{0} \simeq 0.96 a$. The FENE potential does not allow chain scission, but the maximum tensions on covalent bonds for the systems studied here are well below the critical value for scission in breakable-bond models [30].

As a means of varying $N_{e}$, we introduce chain stiffness using the bending potential

$$
U_{\text {bend }}(\theta)=k_{\text {bend }}(1-\cos \theta)
$$

where $\theta$ is the angle betweeen consecutive covalent bond vectors along a chain. Increasing $k_{b e n d}$ increases the rootmean-squared (rms) end-to-end distance of chains $R_{e e}$. The chain stiffness constant $C_{\infty} \equiv<R_{e e}^{2}>/(N-1) l_{0}^{2}$ for well-equilibrated [31] melt states rises from 1.8 to 3.34 as $k_{\text {bend }}$ is increased from 0 to $2.0 u_{0}$. The value of $N_{e}$ decreases from about 70 to about 20 over the same interval [32]. The key parameter in entropic network models is the number of statistical segments per entanglement $N_{e} / C_{\infty}$ (Table @).

The initial simulation cell is a cube of side length $L_{0}$, where $L_{0}$ is greater than the typical end-to-end distance of the chains. $N_{c h}$ chains are placed in the cell, with periodic boundary conditions applied in all three directions. Each initial chain configuration is a random walk of $N-1$ steps with the distribution of bond angles chosen to give the targeted large-scale equilibrium chain structure. In particular the mean value of $\cos (\theta)$ is adjusted so that

$$
C_{\infty}=\frac{1+<\cos (\theta)>}{1-<\cos (\theta)>}
$$


$N_{c h}$ is chosen so that the total number of monomers $N_{\text {tot }}=N N_{c h}$ is $250000\left(L_{0}=66.5 a\right)$ for flexible $\left(k_{\text {bend }}=0\right)$ chains and $70000\left(L_{0}=43.5 a\right)$ for semiflexible $\left(k_{\text {bend }}>0\right)$ chains. The initial monomer number density is $\rho=0.85 a^{-3}$.

After the chains are placed in the cell, we perform molecular dynamics (MD) simulations. Newton's equations of motion are integrated with the velocity-Verlet method [33] and timestep $\delta t=.007 \tau_{L J}-.012 \tau_{L J}$. The system is coupled to a heat bath at temperature $T$ using a Langevin thermostat [34] with damping rate $1.0 / \tau_{L J}$. Only the peculiar velocities are damped.

We first equilibrate the systems thoroughly at $T=$ $1.0 u_{0} / k_{B}$, which is well above the glass transition temperature $T_{g} \simeq 0.35 u_{0} / k_{B}$ [35]. The cutoff radius $r_{c}$ is set to $2^{1 / 6} a$, as is standard in simulations of melts with the bead-spring model [27, 32]. For well-entangled chains, the time required for diffusive equilibration is prohibitively large. To speed equilibration we use the double-bridging-MD hybrid (DBH) algorithm [31], where Monte Carlo moves that alter the connectivity of chain subsections are periodically performed.

Glassy states are obtained from well-equilibrated melts by performing a rapid temperature quench at a cooling rate of $\dot{T}=-2 \times 10^{-3} u_{0} / k_{B} \tau_{L J}$. We increase $r_{c}$ to its final value, typically $1.5 a$, and cool at constant density until the pressure is zero. The quench is then continued at zero pressure using a Nose-Hoover barostat [33] with time constant $10 \tau_{L J}$ until the desired $T$ is reached. Larger values of $r_{c}$ lead to higher final densities and larger stresses at all strains [36], but we have checked that using values of $r_{c}$ as large as $2.6 a$ does not change the conclusions presented below. Indeed, stress-strain curves for different $r_{c} \geq 1.5 a$ collapse when normalized by $\tau_{\text {flow }}[8]$. In this paper $T$ varies from 0 to $0.3 u_{0} / k_{B}$. Simulations at $T=0$ are not directly relevant to experiments, but are useful to gain theoretical understanding of polymer deformation in the limit where thermal activation is not important. To operate in the $T \rightarrow 0$ limit, we remove the Gaussian noise term from the standard Langevin thermostat and retain the viscous drag term.

Values of $N_{e}$ (Table \) are measured by performing primitive path analyses (PPA) [32, 37]. Details of the PPA procedure are the same as those used for undiluted systems in our recent paper [8]. Melt entanglement lengths are consistent with values of $N_{e}$ from the melt plateau moduli [32]. Quenching melt states into a glass has little effect on the values of $N_{e}$ determined from PPA [38]. The changes in entanglement density $\rho_{e}=\rho / 2 N_{e}$ upon cooling are primarily due to a $15 \%$ increase in $\rho$. The conclusion that glasses inherit the melt value of $N_{e}$ is consistent with experimental [39] and simulation [30] studies of the craze extension ratio, as discussed in Section IIA. However, it is inconsistent with some entropic models of strain hardening [3] that assume that $\rho_{e}$ increases rapidly as $T$ decreases.

The values of $N$ employed in this paper are $12-500$ for flexible chains and $4-350$ for semiflexible chains, spanning the range from the unentangled to the fully entangled $\left(N \gg N_{e}\right)$ limits. It is important to note that unentangled systems $\left(N<N_{e}\right)$ are often brittle. This may severely limit the maximum strain that can be studied in experiments and complicate comparison to our simulations.

TABLE I: Chain statistics in fully entangled glasses $\left(N / N_{e}>\right.$ 7)

\begin{tabular}{lccc}
\hline \hline$k_{\text {bend }} a^{2} / u_{0}$ & $N_{e}$ & $C_{\infty}$ & $N_{e} / C_{\infty}$ \\
0.0 & 71 & 1.70 & 42 \\
0.75 & 39 & 2.05 & 19 \\
1.5 & 26 & 2.87 & 9 \\
2.0 & 22 & 3.29 & 7 \\
\hline \hline
\end{tabular}

In fundamental studies of strain hardening [2, 7, 40], compressive rather than tensile deformation is preferred because it suppresses strain localization. This allows the stress to be measured in uniformly strained systems. The rapidity of the quench used here minimizes strain softening, which in turn yields ductile, homogeneous deformation even at the lowest temperatures and highest strains considered.

We employ two forms of compression; uniaxial and plane strain. The stretch $\lambda_{i}$ along direction $i$ is defined as $L_{i} / L_{i}^{0}$, where $L_{i}^{0}$ is the cell side length at the end of the quench. In uniaxial compression, the systems are compressed along one direction, $z$, while maintaining zero stresses along the transverse $(x, y)$ directions [41]. In plane strain compression, the systems are also compressed along the $z$ direction, and zero stress is maintained along the $x$ direction, but the length of the system along the $y$ direction is held fixed $\left(\lambda_{y}=1\right)$.

Compression is performed at constant true strain rate $\dot{\epsilon}=\dot{\lambda}_{z} / \lambda_{z}$, which is the favored protocol for strain hardening experiments [40]. The systems are compressed to true strains of $\epsilon=-1.5$, corresponding to $\lambda_{z}=$ $\exp (-1.5) \simeq 0.22$, for uniaxial compression and $\epsilon=-1.2$ $\left(\lambda_{z} \simeq 0.30\right)$ for plane strain compression. These strains are similar to the highest achievable experimentally [13] in glassy state compression.

Simulations were performed at $\dot{\epsilon}$ between $-10^{-5} / \tau_{L J}$ and $-10^{-3} / \tau_{L J}$. As in previous studies of strain hardening [8] and the initial flow stress [35, 42], a weak logarithmic rise in stress with strain rate was observed for $|\dot{\epsilon}| \lesssim 3 \cdot 10^{-4}$. This small rise does not change the conclusions drawn in the following sections and a similar logarithmic rise is observed in many experiments [43. Thus, while our simulations are performed at much higher strain rates than experiments, we expect that they capture experimental trends. A more rapid change in behavior was observed for $|\dot{\epsilon}| \gtrsim 3 \cdot 10^{-4}$ and qualitative changes in behavior can occur at the much higher rates employed in some previous simulations [25, 26]. For example, the time for stress equilibration across the sample is of order $L_{0} / c_{s}$ where $c_{s}$ is the lowest sound velocity. 
When this time is larger than the time between plastic rearrangements, then each rearrangement occurs before the stress field around it has fully equilibrated in response to surrounding rearrangements. The decorrelated relaxation of different regions leads to a more rapid rise in yield stress with rate [35]. The effect of rate on the relaxation of unentangled chains is discussed in Section IIID.

\section{RESULTS}

\section{A. Comparison to Eight-Chain Model}

The eight chain model [2] has been very successful in describing the functional form of stress-strain curves and is widely used to fit experimental results [28]. It assumes that the entanglement network deforms affinely at constant volume and employs a body centered cubic network geometry with eight chains per node. The stretch of each segment between nodes is then $\lambda_{\text {chain }}=$ $\left(\left(\lambda_{x}^{2}+\lambda_{y}^{2}+\lambda_{z}^{2}\right) / 3\right)^{1 / 2}$, yielding $h=\lambda_{\text {chain }} / \sqrt{N_{e} / C_{\infty}}$ in Eq. 1. This choice of network was the main advance of the eight chain model over previous entropic models $[1,44]$. It allows the model to fit stress-strain curves for various strain states, i.e. shear [4, 5] and uniaxial [2, 3] or plane strain 2, 4] compression [45]. The prediction for the difference between principal stresses along axes $i$ and $j$ in the strain hardening regime is

$$
\sigma_{i}-\sigma_{j}=\tau_{\text {flow }}^{i j}+G_{R} \frac{L^{-1}(h)}{3 h}\left(\lambda_{i}^{2}-\lambda_{j}^{2}\right),
$$

where $\tau_{\text {flow }}^{i j}$ is an independently modeled, rate- and temperature-dependent plastic flow stress [43, 44].

Equation 6] simplifies for the cases of uniaxial and plane strain compression considered here and in many experiments. For uniaxial strain only $\sigma_{z}$ is nonzero and the constant volume constraint implies $\lambda_{x}=\lambda_{y}=\lambda_{z}^{-0.5}$. For plane strain compression, the constant volume constraint requires $\lambda_{x}=\lambda_{z}^{-1}$ and both $\sigma_{z}$ and $\sigma_{y}$ are nonzero. Equation [6 implies a relation between the strain hardening of $\sigma_{z}$ and $\sigma_{y}$, but the latter does not appear to have been measured in experiments.

Despite its wide use, there are fundamental difficulties with the eight chain model that were noted in the Introduction. As a rubber-elasticity based model, it predicts $G_{R}=\rho_{e} k_{B} T$. This prediction is about 100 times too small at $T \sim 0.9 T_{g}$ if values of $\rho_{e}$ are estimated from the melt plateau modulus and has the wrong trend with decreasing $T$ [6, 7]. Some models [3, 46] assume $\rho_{e}$ is much larger than in the melt and rises rapidly as $T$ decreases below $T_{g}$ in order to fit experiments. However, studies of crazing in polymer glasses do not indicate any increase in $\rho_{e}$ over the melt [39, 42]. A constant entanglement density is also consistent with the idea that entanglements represent topological constraints and the observation that the topology does not evolve significantly below $T_{g}$. The extra entanglements added in network models as
$T$ decreases may capture the effect of glassy constraints associated with energy barriers, but it is not clear that it is natural to treat such constraints within a rubberelasticity framework.

Another shortcoming of the eight chain model and more recent work [43, 46] is that the flow stress must be introduced as an independent additive constant. Experiments [47] and our recent simulations [8] suggest that $\tau_{\text {flow }}$ and $G_{R}$ scale in the same way and are controlled by the same physical processes. For example, both decrease nearly linearly as $T$ increases [7, 47], vanish at a strain-rate dependent $T_{g}$, and increase logarithmically with strain rate [48]. Indeed complete strain hardening curves for different rates and cohesion strengths collapsed when scaled by $\tau_{\text {flow }}[8]$. This suggests that $\tau_{\text {flow }}$ is most naturally included as a multiplicative rather than additive factor. To further test this idea we have examined fits to the eight-chain model for a range of $k_{\text {bend }}, T$ and strain states.

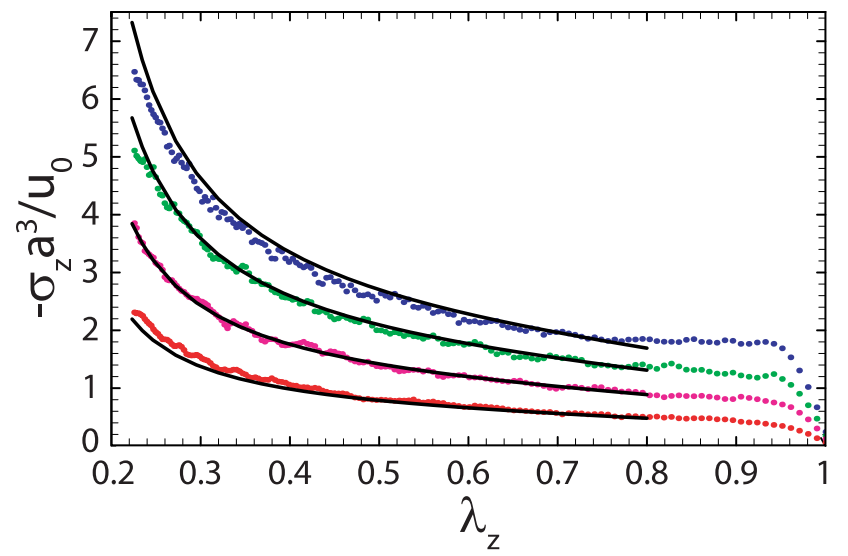

FIG. 1: (Color online) Compressive stress $-\sigma_{z}$ as a function of $\lambda_{z}$ for uniaxial compression at $k_{B} T / u_{0}=0,0.1,0.2$, and 0.3 from top to bottom. The chains had $k_{\text {bend }}=1.5 u_{0}$ and $\dot{\epsilon}=-10^{-4} / \tau_{L J}$. Solid lines show a fit to the eight-chain model (Eq. 6) at $k_{B} T / u_{0}=0.2$ and the extrapolation of this fit to other temperatures using the modified model (Eq. 7). The initial elastic rise and yield for $1>\lambda_{z}>0.8$ are sensitive to aging and are not fit by the eight-chain model.

Figure 1 shows the compressive stress $-\sigma_{z}$ as a function of $\lambda_{z}$ for uniaxial compression of systems with $k_{\text {bend }}=1.5 u_{0}$. Near $\lambda_{z}=1$ there is a sharp elastic increase, followed by yield. Both simulations and experiments 13, 28, 42, 49, 50] find that this initial region $\left(0.8 \lesssim \lambda_{z}<1\right)$ is sensitive to the past history of the sample, including the quench rate and aging. At greater compressions the system is "rejuvenated" and the stress becomes independent of history [13]. Our discussion will focus on this strain hardening regime.

As with experimental data, the strain hardening region $(\lambda<0.8)$ of all curves can be fit (within random stress fluctuations) by adjusting the parameters $\left(\tau_{\text {flow }}^{z x}\right.$, $G_{R}, N_{e}$ ) in Equation 6. The quality of such fits is illustrated for $T=0.2 u_{0} / k_{B}$. Typical uncertainties in fit 
parameters are about $10 \%$. For example, data at all temperatures can be fit with $N_{e}=15 \pm 1$ and best fit values lie within this range. Note that the value of $N_{e}=26$ obtained from the plateau modulus and PPA is substantially larger [32]. Fits of Eq. 6] to experiments [2] also tend to yield smaller values of $N_{e}$ than the plateau modulus.

As in previous work [7, 8, 47], fit values of both the flow stress and hardening modulus decrease linearly with temperature. The temperature where they extrapolate to zero, $T_{g} \simeq 0.41 u_{0} / k_{B}$, is consistent with previous results for the glass transition temperature for this strain rate [35]. Data for all temperatures can be fit with a fixed ratio $\beta \equiv G_{R} / \tau_{\text {flow }}$ anywhere in the range from 0.5 to 0.7. This nearly constant value of $\beta$ provides further support for the idea that strain hardening scales with flow stress.

The above observations can be incorporated into a modified eight chain model that describes the temperature dependent strain hardening in terms of only four parameters $\tau_{\text {flow }}^{0}, T_{g}, N_{e}$ and a temperature independent ratio $\beta$. Here $\tau_{\text {flow }}^{0}$ is the flow stress at $T=0$ and at other temperatures $\tau_{\text {flow }}=\tau_{\text {flow }}^{0}\left(1-T / T_{g}\right)$. The shear stress in the strain hardening regime is written as

$$
\sigma_{i}-\sigma_{j}=\tau_{\text {flow }}^{0, i j}\left[1-\frac{T}{T_{g}}\right]\left[1+\beta \frac{L^{-1}(h)}{3 h}\left(\lambda_{i}^{2}-\lambda_{j}^{2}\right)\right] .
$$

Note that Eq. 7 is equivalent to the usual eight-chain model except that it imposes proportionality between the flow stress and hardening modulus and assumes a linear temperature drop in both. This reduction in parameters may be useful in extrapolating experimental data, since values for one temperature determine those at any other if $T_{g}$ is known.

The solid lines in Figure 1 show predictions of Eq. 7 based on the fit at $T=0.2 u_{0} / k_{B}: \tau_{\text {flow }}^{0}=0.634 u_{0} a^{-3}$, $\beta=0.56$, and $N_{e}=14.1$. The predictions agree quite well with simulation results over an extremely wide range of $T / T_{g}$. The largest deviations are of order $10 \%$ at $T=0$ and the smallest $\lambda_{z}$. There is a slight over-prediction of the change in curvature with increasing $T$ that manifests as slight $(\sim 10 \%)$ increases in $\beta$ or decreases in $N_{e}$ in best fits to the data, particularly at $T=0.3$. Fits of the same quality are obtained for all $k_{\text {bend }}$ and strain states (see Fig. 2), suggesting that this simple extrapolation may be widely applicable to data from simulations or experiment.

A more stringent test of Eq. 7 is whether it is able to predict stresses for multiple strain states with the same parameters. As pointed out by Arruda and Boyce [2], uniaxial and plane strain compression produce extremely different changes in chain configuration. Under plane strain compression the chains all stretch in one direction, while in uniaxial compression the chains stretch along all directions in the plane perpendicular to the compression axis.

Figure 2 shows results for plane strain compression of the same systems as Fig. 1. As before, excellent fits can be obtained to Eq. 6] and extrapolations from fits at one temperature using Eq. 7 capture the variation in stress over the full temperature range. Despite these successes, there are troubling inconsistencies in the parameters of these fits. While the values of $G_{R}$ for $\sigma_{z}$ in plane strain and uniaxial compression are consistent (within our $10 \%$ uncertainty), the value of $N_{e}$ is significantly higher for plane strain; $N_{e}=20 \pm 2$. In addition, the strain hardening of $\sigma_{y}$ and $\sigma_{z}$ in plane strain are inconsistent. From Eq. 6. the value of $\sigma_{y}$ is determined up to an additive constant from measurements of $\sigma_{z}$. The dashed line in Fig. 2(b) shows this prediction for $T=0$ with the additive constant adjusted to fit data at large $\lambda_{z}$. At all temperatures the variation in $\sigma_{y}$ is systematically smaller than predicted from $\sigma_{z}$. The decrease corresponds to a reduction in $G_{R}$ for $\sigma_{y}$ by about $20 \%$. In contrast, best fit values of $N_{e}$ for $\sigma_{y}$ and $\sigma_{z}$ match to within $1 \%$.

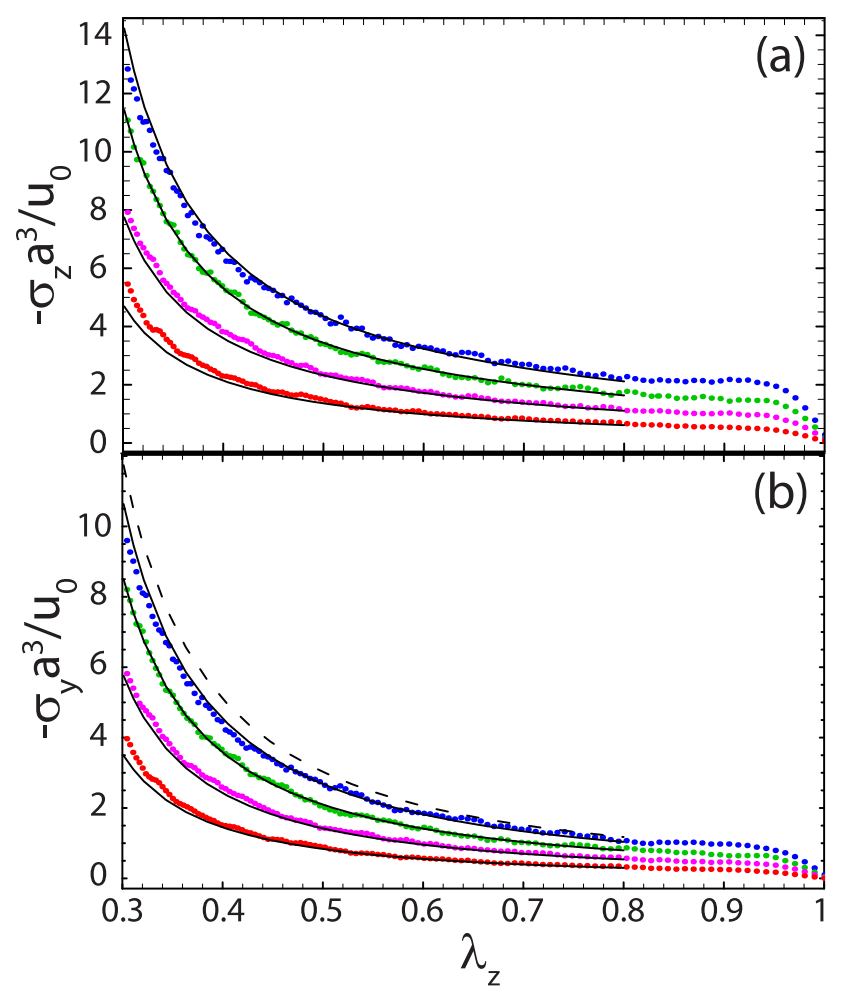

FIG. 2: (a) Compressive stress $-\sigma_{z}$ and (b) perpendicular stress $-\sigma_{y}$ as a function of $\lambda_{z}$ for plane strain compression at $k_{B} T / u_{0}=0,0.1,0.2$, and 0.3 from top to bottom in each panel. The chains had $k_{\text {bend }}=1.5 u_{0}$ and $\dot{\epsilon}=-10^{-4} / \tau_{L J}$. Solid lines show a fit to the eight-chain model (Eq. 6) at $k_{B} T / u_{0}=0.1$ and the extrapolation of this fit to other temperatures using the modified model (Eq. 7). The dashed line in (b) shows the variation in $\sigma_{y}$ predicted by Eq. 6] and measured values of $\sigma_{z}$.

A strong dependence of model parameters such as $G_{R}$ and $N_{e}$ on strain state has also been noted experimentally [9]. One possibility is that the strain state changes the role of entanglements in ways that are not captured completely by the eight-chain model. Another is that while the eight-chain model provides a useful fitting function, 
it does not capture the correct strain hardening mechanisms. In this case, fit parameters may not have direct physical significance. The scaling of $G_{R}$ with $\sigma_{\text {flow }}$ rather than $T$ supports the view that entropy is not the dominant source of stress and this is examined further below. It is also important to note that the fits above assumed constant volume, but the simulation volume decreased with strain by up to $8 \%$ for plane strain and large $k_{\text {bend }}$. Including the correct $\lambda_{i}$ in Eq. 6 would change the predicted stress by up to $15 \%$. In the following section we show that violations of the assumption of affine displacement of entanglements can produce similar changes. Thus the fit parameters compensate for changes that are not included in the model, further reducing their direct relevance.

\section{B. Dissipative and Energetic Stresses}

Entropic network models assume that strain hardening arises entirely from a reversible increase in the entropy of the entanglement network. Experiments showed many years ago that strain hardening is also associated with large increases in internal energy [13], but this observation has not been incorporated into published theoretical models. Simulations allow us to separate the role of entropy and energy in strain hardening.

For uniaxial and plane strain compression, the stress along the compressive axis is directly related to the work $W$ done on the system per unit strain: $\sigma_{z}=\partial W / \partial \epsilon_{z} \cdot \sigma_{z}$ can be separated into an energetic component $\sigma_{z}^{U}$ and a thermal component $\sigma_{z}^{Q}$ using the first law of thermodynamics: $d W=d Q+d U$, where $U$ is the internal energy of the system and $d Q$ is the heat transfer away from the system. This implies

$$
\sigma_{z}=\frac{\partial W}{\partial \epsilon_{z}}: \sigma_{z}^{U}=\frac{\partial U}{\partial \epsilon_{z}}: \sigma_{z}^{Q}=\frac{\partial Q}{\partial \epsilon_{z}}=\sigma_{z}-\sigma_{z}^{U} .
$$

These quantities are readily obtained from our simulation data and could in principle be obtained by differentiating results for $W$ and $Q$ from deformation calorimetry experiments. Unfortunately existing studies [51, 52, 53] have not extended into the strain hardening regime.

Experimental data are frequently plotted in a manner designed to isolate the Gaussian and Langevin contributions to the strain (Eq. 1). If $\sigma_{z}$ is plotted against $g(\bar{\lambda})=\lambda_{z}^{2}-\lambda_{x}^{2}$, then Eq. 6 predicts a straight line in the Gaussian limit $(h<<1)$. The Langevin correction $\left((3 h)^{-1} L^{-1}(h)\right)$ adds an upwards curvature. Since $\lambda_{x}$ is not generally measured, experimental stresses are plotted as a function $g\left(\lambda_{z}\right)$ that is determined by assuming constant volume. For uniaxial strain $g\left(\lambda_{z}\right)=\lambda_{z}^{2}-1 / \lambda_{z}$ and for plane strain $g\left(\lambda_{z}\right)=\lambda_{z}^{2}-1 / \lambda_{z}^{2}$.

Figure 3 illustrates the variation of total, thermal and energetic stresses with $g$ under uniaxial and plane strain compression for the most highly entangled system $\left(N_{e}=22\right)$. The total stress for both strain states shows strong upward curvature that is normally attributed to the Langevin correction. As expected from entropic network models, the amount of curvature decreases with increasing $N_{e}$ [8]. However, Fig. 3 shows that this curvature is not related to entropy. Almost all of the upward curvature is associated with the energetic contribution to the stress, while $\sigma_{z}^{Q}$ shows the linear behavior expected for Gaussian chains. Similar behavior is observed for all $N_{e}$ and $T$, and we now discuss the trends in $\sigma^{Q}$ and $\sigma^{U}$ separately.
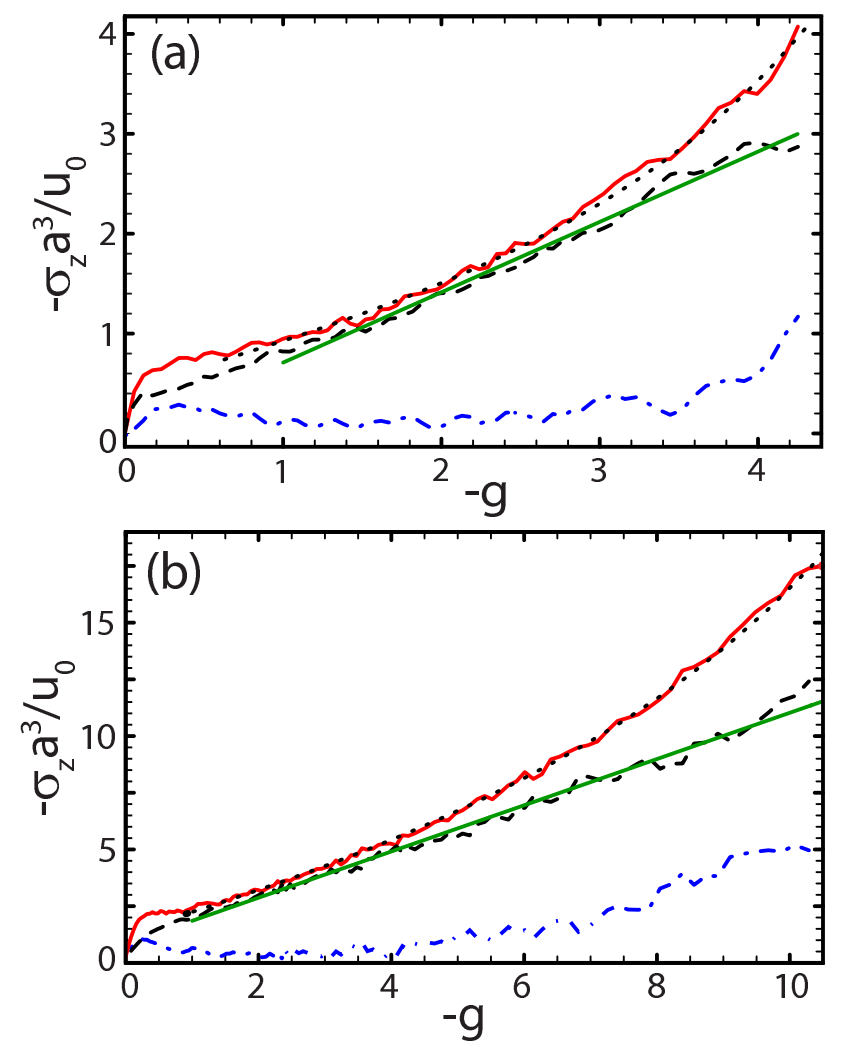

FIG. 3: (Color online) Total stress (solid lines) and thermal (dashed lines) and potential energy (dot-dashed lines) contributions for (a) uniaxial compression at $k_{B} T / u_{0}=0.2$ and (b) plane strain compression at $k_{B} T / u_{0}=0$. The systems had $k_{\text {bend }}=2.0\left(N_{e}=22\right), N=350$ and (a) $\dot{\epsilon}=-10^{-5} / \tau_{L J}$ or (b) $\dot{\epsilon}=-10^{-4} / \tau_{L J}$. Dotted lines show best fits of $\sigma_{z}$ to Eq. 7 with (a) $N_{e}=15.5$ and (b) $N_{e}=22$. Straight lines are fits to $\sigma_{z}^{Q}$. Both $\sigma_{z}$ and $g$ are negative under compression.

For all systems, temperatures, and strain states the thermal stress is well fit by the linear behavior expected for Gaussian chains. There may be a small upwards curvature, particularly for uniaxial compression, but it is comparable to statistical fluctuations. Attempts to fit $\sigma_{z}^{Q}$ to the eight chain model always require increasing $N_{e}$ significantly above values obtained from the melt plateau modulus.

We define a thermal hardening modulus $G_{\text {therm }}$ from the slope of linear fits to $\sigma_{z}^{Q}=\sigma_{0}+G_{\text {therm }} g\left(\lambda_{z}\right)$. Table III shows values for $G_{\text {therm }}$ for uniaxial and plane strain compression for various entanglement lengths at $T=0.2 u_{0} / k_{B}$. While $G_{\text {therm }}$ is systematically higher 
for uniaxial compression, the differences (10-30\%) are not large. In contrast, $G_{\text {therm }}$ decreases rapidly with increasing $N_{e}$. Rubber elasticity theories for Gaussian chains would predict $G_{R} \propto N_{e}^{-1}$ and it is interesting that $G_{\text {therm }}$ appears to scale in this way. As shown in Table II changes in $N_{e} G_{t h e r m}$ are within our statistical error bars $(\sim 10 \%)$ and show no systematic trend with $N_{e}$.

TABLE II: Thermal Moduli $G_{\text {therm }}$ in units of $u_{0} / a^{3}$ for $T=$ $0.2 u_{0} / k_{B}$ and $\dot{\epsilon}=-10^{-4} / \tau_{L J}$. Errorbars are about $10 \%$.

\begin{tabular}{lcccc}
\hline \hline$N_{e}$ & $G_{\text {therm }}^{\text {uniax }}$ & $G_{\text {therm }}^{\text {plane }}$ & $N_{e} G_{\text {therm }}^{\text {uniax }}$ & $N_{e} G_{\text {therm }}^{\text {plane }}$ \\
22 & 1.3 & 1.0 & 28 & 23 \\
26 & 1.0 & 0.75 & 27 & 20 \\
39 & 0.57 & 0.50 & 22 & 20 \\
71 & 0.37 & 0.34 & 26 & 24 \\
\hline \hline
\end{tabular}

Figure 4 shows $\sigma_{z}^{U}$ during plane strain compression for different $N_{e}$. Results are shown for $T=0$ because the energetic stresses are the largest, but results at higher temperatures show similar trends. The value of $\sigma^{U}$ rises to a peak near the yield point, and then drops to a nearly constant value for $|g|>1$. The initial behavior for $|g|<$ 1 is nearly independent of the entanglement length but does depend weakly on age and strain rate. The behavior at slightly larger $|g|$ depends strongly on entanglement length (Fig. (4). For example, at $T=0.2 u_{0} / k_{B}$ the ratio of the constant energetic stress to the flow stress rises from about $4 \%$ for flexible chains to $16 \%$ for the most entangled system. Similar ratios are obtained for the $T=0$ data in Fig. 4 .

Hasan and Boyce examined the enthalpy stored in samples of polystyrene (PS), polymethylmethacrylate (PMMA) and polycarbonate (PC) as a function of residual strain [13]. They found a sharp increase in enthalpy up to a strain of about $20 \%(|g|=0.55)$ that was larger in annealed samples than in rapidly quenched samples like those used here. For quenched PS, the magnitude of the rise in energy density is about $4 \mathrm{MPa}$. Values for the work performed are difficult to extract from the paper, but as a rough estimate we take the flow stress (55MPa) times the strain (0.2) and find 11MPa. Thus in the initial stages of deformation, of order a third of the work is stored in energy. Calculating the same ratio for our simulations gives values between 30 and $45 \%$.

For strains from -0.2 to $-0.8(|g|=2.0)$ or larger, Hasan and Boyce found a weak, nearly linear rise in enthalpy. This corresponds to a constant $\sigma^{U}$ like that observed in Fig. 4 for intermediate $|g|$. Analysis of their figures 13] shows that the ratio of $\sigma^{U}$ to the flow stress increases from about $4 \%$ for PS to $15 \%$ for PC. Since PC is more entangled than PS, this trend is the same as observed in Fig. 4. Note that in both simulations and experiments the fraction of work stored as energy depends strongly on the strain amplitude. The fraction stored during the initial rise to the flow stress is dependent on sample age
[13] and may be of order $50 \%$ or more [54, 55]. As $|g|$ increases and $\sigma^{U}$ remains constant, the fraction stored as energy drops towards the much smaller value given by $\sigma^{U} / \sigma_{\text {flow }}$.

Fig. 4 shows a sharp rise in $\sigma^{U}$ at the largest values of $|g|$. The onset moves to smaller $|g|$ and the magnitude of the rise increases as $N_{e}$ decreases. This rise is the source of almost all the curvature in the total stress. The experiments of Hasan and Boyce [13] did not show this rise. One reason may be that their experiments only extended to uniaxial strains of $-0.8(|g|=2.0)$ for the most entangled systems. However, their measurement is also limited to the residual enthalpy after the sample is unloaded. Simulations were performed to determine the amount of energy recovered during unloading from different strains. The recovered energy is relatively small in the region where $\sigma^{U}$ has a small constant value, but rises rapidly at larger strains. Indeed, almost all of the energy that contributes to the sharp rise in $\sigma^{U}$ at large $|g|$ is recovered when samples are unloaded. Thus experiments could only observe this rise by using deformation calorimetry.

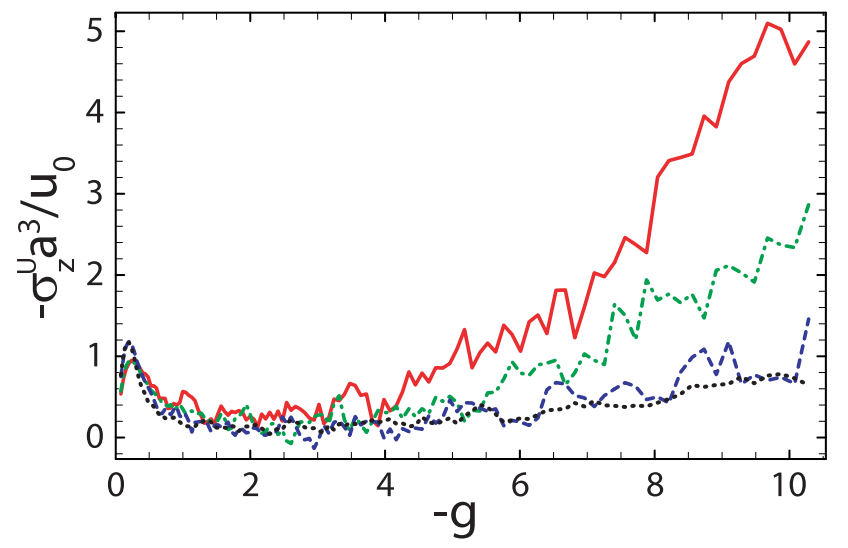

FIG. 4: (Color online) Energetic components of stress $\sigma_{z}^{U}$ for plane strain compression at $T=0$ with strain rate $\dot{\epsilon}=$ $-10^{-4} / \tau_{L J}$ for $N_{e}=22$ (solid line), $N_{e}=26$ (dot-dashed line), $N_{e}=39$ (dashed line), and $N_{e}=71$ (dotted line).

The rise in energetic stress at large $|g|$ seems to occur when segments of length $N_{e}$ are pulled taut between entanglements. To demonstrate this we examined changes in chain statistics with stretch. Entropic network models assume that the deformation of the entanglement network is affine to the macroscopic stretch. An affine displacement would, on average, increase the length of any chain segment by a factor of $\lambda_{\text {chain }}$. This stretch can not apply at the smallest scales since the length of chemical bonds $l_{0}$ can not increase significantly. As a result chains are pulled taut and deform subaffinely on small scales. The larger the strain, the larger the length of taut segments.

To illustrate this we compare the rms Euclidean distance between monomers separated by $\mathrm{n}$ bonds $R(n)$ to the affine prediction $R_{a f f}(n)$. If $R_{0}(n)$ is the distance 
before stretching, then $R_{a f f}(n)=\lambda_{\text {chain }} R_{0}(n)$ where $\lambda_{\text {chain }}^{2}=\left(\lambda_{x}^{2}+\lambda_{y}^{2}+\lambda_{z}^{2}\right) / 3$. For the case of plane strain, $\lambda_{\text {chain }}^{2}=\left(\lambda_{z}^{2}+1+\lambda_{z}^{-2}\left(V / V_{0}\right)^{2}\right) / 3$, where $\lambda_{x}$ has been eliminated by using the ratio $V / V_{0}$ of the final and initial volumes. Volume changes are normally ignored, but are large enough to affect the plots shown below.

Figure 5 (a) shows the ratio of the observed $R(n)$ to the affine prediction as a function of $n / N_{e}$ for different $g\left(\lambda_{z}\right)$ and $N_{e}$ under plane strain. There is a clear crossover from subaffine behavior $\left(R / R_{a f f}<1\right)$ to affine behavior $\left(R / R_{a f f} \simeq 1\right)$ with increasing $n$. In the subaffine regime at small $n$, chains are pulled nearly taut. The crossover to affine behavior moves to larger $n$ as $|g|$ increases, implying that chains are pulled straight over longer segments. For chains with $N_{e}=39$ the crossover remains slightly below $N_{e}$ at the largest strains considered here. However for $N_{e}=22$ the crossover appears to reach $N_{e}$ by $|g|=5$. At larger $|g|$ the magnitude of $R / R_{a f f}$ decreases, but the region of rapid crossover appears to remain near $N_{e}$. This suggests that the entanglements prevent chains from stretching taut on longer scales.

Figure 5(b) shows there is a direct correlation between subaffine deformation at $N_{e}$ and the increase in the energetic contribution to the stress. The values of $\sigma_{z}^{U}$ from Fig. 4 are replotted against $R\left(N_{e}\right) / R_{a f f}\left(N_{e}\right)$ instead of $|g|$ [56]. There is a sharp rise in $\sigma_{z}^{U}$ as $R\left(N_{e}\right) / R_{a f f}\left(N_{e}\right)$ decreases below about 0.925 . As seen in panel (a), this corresponds roughly to the point where the length of taut segments reaches $N_{e}$. This suggests that the energetic stress arises when the entanglement network begins to resist further deformation. As expected from this picture, we find a growing tension in covalent bonds as $\sigma_{z}^{U}$ rises. However, the maximum tensions in the "worst case" scenario of plane strain compression for $N_{e}=22$ are only about $100 u_{0} / a$, which is well below the breaking strength $240 u_{0} / a$ used in breakable-bond simulations [30].

The above findings help to explain some of the discrepancies in the fit parameters for the eight-chain model. The upwards curvature in plots of $\sigma_{z}$ vs. $|g|$ comes from energetic terms rather than entropy. Fits to uniaxial and plane strain give different $N_{e}$ because the energetic contributions are different. However the fit values are never far from $N_{e}$ because the sharp increase in $\sigma_{z}^{U}$ occurs when segments of length $N_{e}$ are pulled taut. Fig. 5(a) also indicates that the entanglement network does not deform completely affinely as assumed in the eight-chain model. Even at small strains $R\left(N_{e}\right) / R_{a f f}\left(N_{e}\right)$ is slightly less than one and this would produce significant $(\sim 10 \%)$ changes in the stress from Eqs. 6 or 7 [57].

Note that the deviations from affinity observed in Fig. 5] are significantly smaller than those predicted from rubber-elasticity based models for the non-affine deformations in entangled polymers above $T_{g}[58,59]$. These models assume that fluctuations about affine deformation are confined to the "tube" formed by surrounding entanglements. They predict nonaffine reductions in $R\left(N_{e}\right) / R_{a f f}\left(N_{e}\right)$ that are about $50 \%$ greater than our results at small strains. At the larger strains where we find entanglements produce a significant energetic stress, the disparity decreases. The discrepancy appears to reflect the fact that the nonaffine displacements in our simulations [8] are much smaller than the tube radius until the energetic stress begins to dominate. Interestingly, the magnitude of the nonaffine displacements decreases slightly with increasing $r_{c}$, suggesting they are limited by cohesive interchain interactions rather than entanglements. These observations provide further evidence that polymers in a glass are not free to explore their tube as assumed in entropic models. It would be interesting to extend these comparisons to melt models to see what additional information can be obtained.
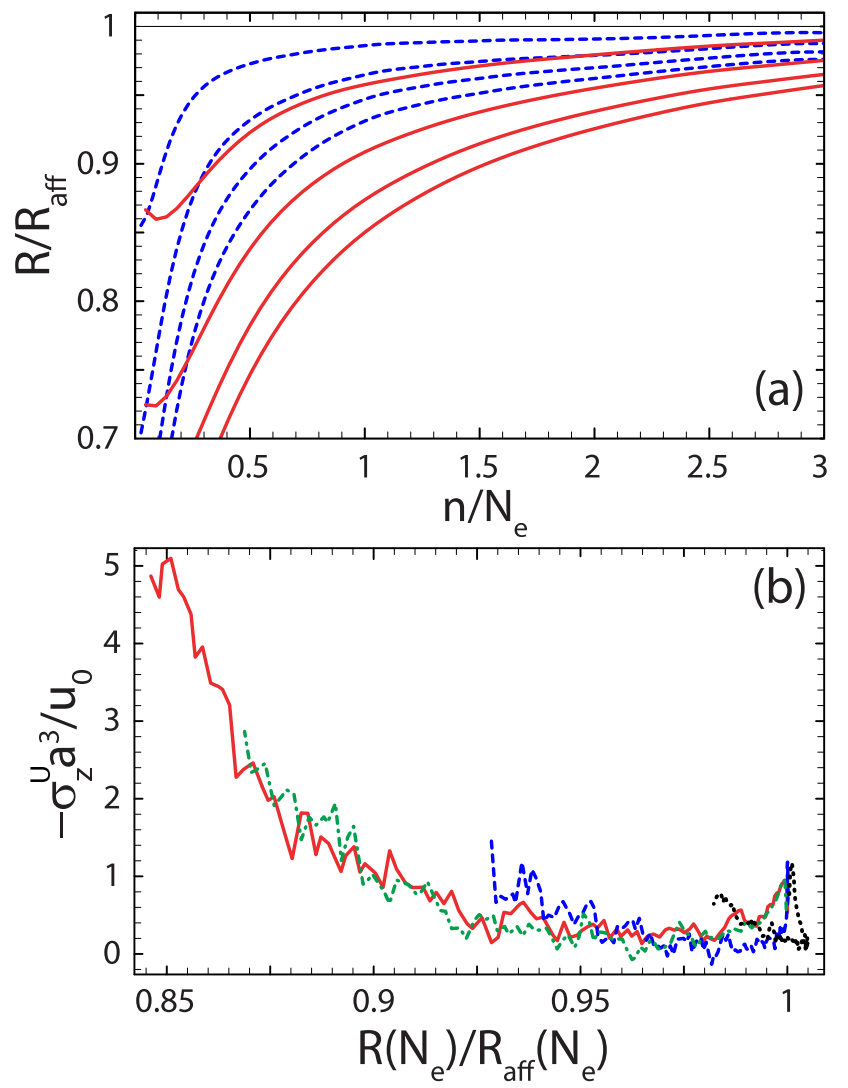

FIG. 5: (Color online) (a) $R(n) / R_{a f f}(n)$ for the same systems and conditions as Fig. 4 with $n$ scaled by $N_{e}$. Solid lines indicate $N_{e}=22$ and dashed lines indicate $N_{e}=39$. Curves are for $|g|=2.5,5,7.5$, and 10 from top to bottom. (b) $\sigma_{z}^{U}$ plotted against $R / R_{a f f}$ evaluated at $n=N_{e}$. This corresponds to evaluating $\sigma_{z}^{U}$ along a vertical slice of (a). Solid, dot-dashed, dashed, and dotted lines indicate data for $N_{e}=22,26,39$, and 71 respectively [56].

\section{Reversibility and entropic back stresses}

If the work performed in deforming a glass is entropic, it should be reversible. However, large strain experiments performed well below $T_{g}$ show only $\sim 10 \%$ strain recovery upon unloading [13]. Entropic network models postulate 
that there is an entropic "back stress" [2] that favors further strain reduction but that relaxation is too slow to observe because of the high viscosity of the glassy state [1]. As expected from this picture, lowering the viscosity by heating even slightly above $T_{g}$ leads to nearly complete shape recovery of well-entangled glasses [28].

To see if similar behavior occurs in simulations, we loaded samples to $\epsilon=-1.5$ at $\dot{\epsilon}=-10^{-5} / \tau_{L J}$ and $T=$ $0.2 u_{0} / k_{B}$. The samples were then unloaded at the same $|\dot{\epsilon}|$ and $T$ until all $\sigma_{i}$ were zero. Fully entangled samples $\left(N=350, N_{e}=39\right)$ recovered only $6 \%$ of the peak strain and unentangled chains $(N=16)$ recovered slightly less, $\sim 4 \%$. The result for entangled chains is comparable to experiments [13].

The samples were then heated to $T=0.4 u_{0} / k_{B}$ over $100 \tau_{L J}$ and allowed to relax with a Nose-Hoover barostat imposing zero stress in all three directions. Figure 6(a) shows the resulting strain recovery. For the entangled system, an additional $87 \%$ of the strain was recovered after $10^{5} \tau_{L J}$ and the rate of recovery remained significant at the end of this period. In the unentangled system, $46 \%$ of the strain is recovered, mainly in the first $2 \times 10^{4} \tau_{L J}$. While this recovery is substantially smaller than for entangled systems, network models would predict no strain recovery for unentangled chains. Examination of pair and bond energies shows that they are nearly constant during the relaxation at $T=0.4 u_{0} / k_{B}$. These results imply that entropic stresses drive the relaxation and that entanglements play an important role.

To monitor the entropy in chain confirmations we evaluated the orientational order parameter, $P_{2}(\cos (\alpha))=$ $\left(3 \cos ^{2}(\alpha)-1\right) / 2$, where $\cos ^{2}(\alpha)=<R_{z}^{2}>/<R_{e e}^{2}>$. This quantity measures the deviation from isotropy at the end-end scale. There is significant orientation of both short and long chains during the initial strain, which is discussed further below. During the relaxation to zero stress and heating to $T=0.4 u_{0} / k_{B}$ the orientation relaxes only $3 \%$ for entangled chains and $5 \%$ for unentangled chains. As shown in Fig. 6(b), rapid and substantial deorientation occurs during the strain relaxation above $T_{g}$. Short chains become nearly isotropic after only $\sim 2 \cdot 10^{4} \tau_{L J}$, and there is little strain recovery after this point. Entangled chains deorient more slowly, and both $P_{2}$ and $\epsilon_{z}$ are continuing to evolve slowly at the end of the simulations. These results clearly show that the entropy of chain orientation drives the strain relaxation. They also show that the network of entangled chains prevents chains from deorienting without recovery of the macroscopic strain.

While Fig. 6 provides strong support for an entropic back stress, the magnitude of this stress can only be of order $\rho_{e} k_{B} T_{g}$ and thus much smaller than the stresses associated with strain hardening. To confirm this we took the $N=350$ sample studied in Fig. 6 and heated to $T=0.4 u_{0} / k_{B}$ with different stress control. Instead of fixing all $\sigma_{i}$ to zero, only the total pressure $p=-\left(\sigma_{x}+\sigma_{y}+\sigma_{z}\right) / 3$ was kept at zero while the ratios of the $L_{i}$ were fixed at the values after deformation. After

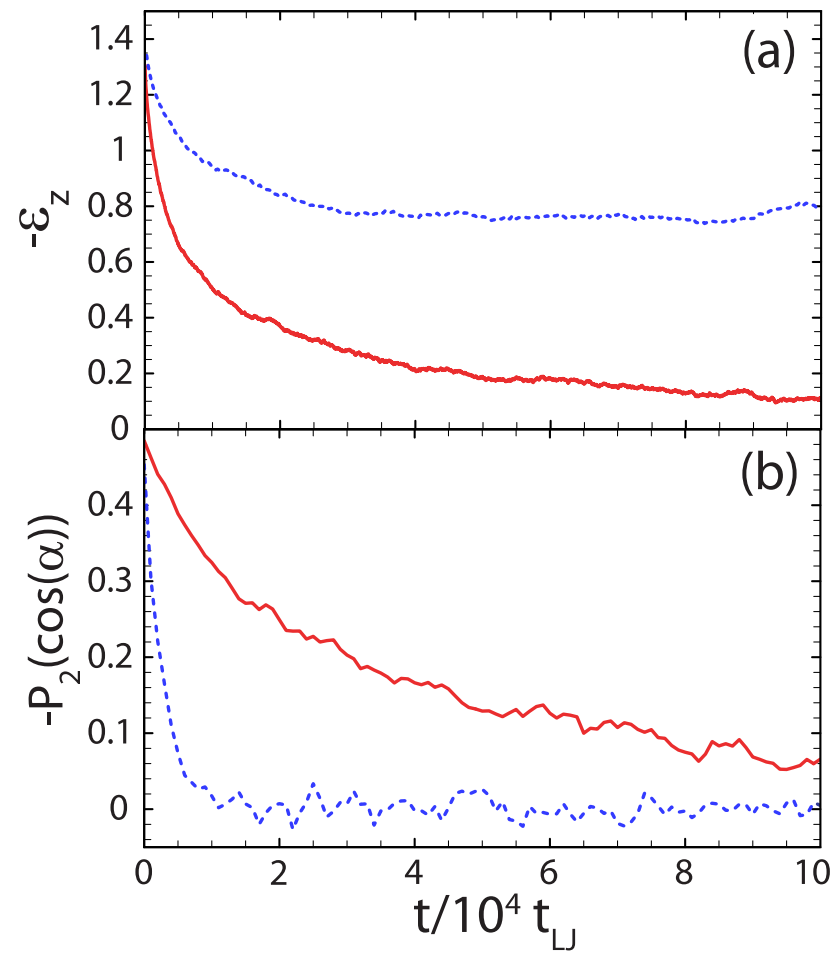

FIG. 6: (Color online) Time dependent relaxation at $T=$ $0.4 u_{0} / k_{B}$ of (a) true strain and (b) chain orientation parameter $P_{2}$ for entangled $N=350$ (solid lines) and unentangled $N=16$ (dashed lines) systems. Systems were prepared by loading to $\epsilon_{z}=1.5$ at $T=0.2 u_{0} / k_{B}$, unloading to zero stress and then heating to $0.4 u_{0} / k_{B}$ over $100 \tau_{L J}$.

heating, there was a shear stress $\sigma_{z}-\sigma_{x} \approx \sigma_{z}-\sigma_{y}$ whose direction favored relaxation back to $\epsilon=0$. The magnitude of this stress relaxed rapidly $\left(\sim 10^{4} \tau_{L J}\right)$ to about twice the entropic estimate of $\rho_{e} k_{B} T_{g} \approx 0.02 u_{0} / a^{3}$, while the stress during strain hardening below $T_{g}$ is more than two orders of magnitude larger. Similar results were obtained at higher temperatures. We next applied a shear force of the same magnitude $\left(0.04 u_{0} / a^{3}\right)$ to an unstrained system at $T=0.4 u_{0} / k_{B}$. The magnitude of the strain produced by this stress over $10^{5} \tau_{L J}$ was 1.2 , which is comparable to that during stress relaxation (Fig. 6). Both this driven response and the stress relaxation varied approximately as the logarithm of time, indicating that the sample displays creep rather than viscous flow. Recent studies of a similar glassy system also show creep behavior at this temperature and time scale [60].

\section{Chain Length Effects}

The orientation of unentangled chains shown in Fig. 6(b) is not expected from entropic network models. For $N<N_{e}$ there is no entanglement network spanning the system. Network models assume that this network is essential in forcing the deformation of individual chains to follow the macroscopic strain. However because chains 
are not free to relax in the glassy state, chain orientation can occur even without entanglements. In recent work on uniaxial compression, we found significant strain hardening of unentangled chains [8] and discovered a direct connection to chain orientation [17] as suggested by recent analytic studies [29]. In this section we extend the study of chain length dependence to other strain states and systems.

Figure 7(a) shows stress-strain curves for flexible chains $\left(N_{e}=71\right)$ in plane strain compression for a range of $N$ between 12 and 500. At small $|g|$, the stresses are nearly independent of $N$. Beyond yield, the stresses increase faster for larger $N$, reaching an asymptotic limit for $N \gg N_{e}$ as expected from network models [8]. However, there is significant strain hardening for chains as short as $N_{e} / 6$. Similar behavior is observed for all entanglement densities under both uniaxial and plane strain. This is illustrated for $k_{\text {bend }}=2.0 u_{0}\left(N_{e}=22\right)$ under uniaxial strain in Fig. 10(a) and for $k_{\text {bend }}=0.75 u_{0}$ in Fig. 2(c) of Ref. [17].

Examination of individual chain conformations shows that strain hardening is directly correlated with increasing chain orientation [61]. To quantify this we define a microscopic stretch of chains as $\lambda_{i}^{c} \equiv R_{i} / R_{i}^{0}$ where $R_{i}$ is the rms projection along $i$ of the end-end distance and $R_{i}^{0}$ is the value before deformation. Fig. 7 shows $\lambda_{i}^{c}$ for flexible chains under plane strain compression. For fully entangled chains $\left(N / N_{e} \sim 7\right)$ the microscopic stretch remains close to the macroscopic stretch as already concluded from Fig. 5. For $\lambda_{x}$ the deviation is smaller than the line width. For $\lambda_{z}$ the maximum deviations of about $2 \%$ (lowest dashed line in Fig. 7(c)) can be attributed to non-affine deformation of the unentangled ends of the chains.

As the chain length decreases, the microscopic stretch shows increasing deviations from the macroscopic stretch. Chains compress by less than the imposed strain along the $z$ axis, and stretch by a smaller amount along the $x$ axis. For each $N, \lambda_{i}^{c}$ is close to the entangled results at small $|g|$ and then saturates at large $|g|$. The onset of saturation in $\lambda_{i}^{c}$ correlates with the saturation of the stress, and moves to larger $|g|$ with increasing $N$. These results clearly show that entanglements force the chain orientation to follow the macroscopic stretch but that significant chain orientation occurs without entanglements. Strain also orients chains in unentangled melts, but is only appreciable when the strain rate is faster than chain relaxation times [62]. The extremely slow dynamics in glasses prevents relaxation of shear-induced orientation.

Fig. 7(d) shows the ratio of the product of the chain and macroscopic stretches $\Pi_{i} \lambda_{i}^{c} / \Pi_{i} \lambda_{i}$. This corresponds to the ratio of changes in the volume subtended by the chains to changes in the macroscopic volume. For entangled chains the ratio is close to unity, as expected for a crosslinked network. The volume subtended by unentangled chains need not follow the macroscopic volume, but the observed deviations are less than $11 \%$ in Fig. [7(d).
Deviations are even smaller for flexible chains under uniaxial strain.

Figure 8 shows that $\sigma_{z}$ is determined directly by the microscopic orientation of chains rather than the macroscopic deformation. Results for plane strain compression of flexible chains (from Fig. 7(a)) and uniaxial compression of semiflexible chains $\left(N_{e}=39\right)$ are plotted against an effective $g$ calculated from $\lambda_{i}^{c}: g_{\text {eff }} \equiv\left(\lambda_{z}^{c}\right)^{2}-\left(\lambda_{x}^{c}\right)^{2}$. When plotted against this measure of microscopic chain orientation, results for all chain lengths collapse onto a universal curve. A similar collapse was obtained in Ref. [17] using a single effective orientation parameter $\lambda_{z}^{e f f}$ along the compression direction. This was obtained by measuring $\lambda_{x}^{c}$ and using the assumption of constant chain volume to determine $\lambda_{z}^{e f f}$ (i.e. $\lambda_{z}^{\text {eff }}=1 / \lambda_{x}^{c}$ for plane strain). The collapse produced for $g\left(\lambda_{z}^{e f f}\right)$ is nearly identical to that in Fig. 8 because chain volume is nearly constant (Fig. 7(d)) and $g_{\text {eff }}$ is mainly determined by $\lambda_{x}$

Fig. 9 shows that results for $\sigma_{z}-\sigma_{y}$ during plane-strain compression also depend only on microscopic chain orientation. When plotted against the macroscopic $|g|$, results for unentangled chains lie substantially below those for entangled chains. When plotted instead against the microscopic orientation function $g_{\text {eff }}=\left(\lambda_{z}^{c}\right)^{2}-\left(\lambda_{y}^{c}\right)^{2}$, data for all chains collapse onto a universal curve (Fig. 9(b)). Note that $\lambda_{y}^{c}$ decreases by as much as $5 \%$ from $\lambda_{y}=1$ for the shortest chains, and this affects the data collapse 63 .

The quality of the collapse of the total stress decreases slightly as the entanglement length decreases. This is illustrated for $N_{e}=22\left(k_{\text {bend }}=2.0\right)$ in Fig. 10. Results for fully entangled systems $\left(N \geq 4 N_{e}\right)$ collapse completely. Data for smaller $N$ follow the asymptotic curve at small $\left|g_{\text {eff }}\right|$, and then drop below it at a $\left|g_{\text {eff }}\right|$ that decreases with decreasing $N$. The smallest chains in Fig. 10 and 8(b) are only a few persistence lengths and may not behave like Gaussian chains [17]. However such effects are not large enough to explain why results for short chains fall below the asymptotic curve in Fig. $10(b)$.

These discrepancies are instead explained by examining the variation with $N$ of the energetic contribution to the stress. Figure 10(c) shows $\sigma_{z}^{U}$ plotted against $g\left(\lambda_{e f f}\right)$. The initial peak at low $\left|g_{e f f}\right|$ is nearly independent of $N$, but the behavior at large $\left|g_{e f f}\right|$ is not. There is a sharp rise in $\sigma_{z}^{U}$ for fully entangled chains, that does not occur for $N \leq 44$. The magnitude of this rise is comparable to the deviation between results for $N=44$ and the asymptotic curve for entangled chains in Fig. 10(a). These results suggest that while the thermal contribution to the stress depends only on the chain orientation, the energetic contribution at large $|g|$ only occurs for entangled chains. Without the entanglement network, chains can contract along their tube to eliminate the large energetic stresses.

We have confirmed that increasing the strain rate from $|\dot{\epsilon}|=10^{-5} / \tau_{L J}$ to $10^{-3} / \tau_{L J}$ does not change the relation 
between chain orientation and stress described in this section. The main effect is to increase $\lambda_{e f f}$ towards $\lambda$ with the increase being more pronounced for shorter chains. At even higher strain rates, there is almost no relaxation, and $\lambda_{\text {eff }}$ is close to $\lambda$ for all $N$. This is the regime observed in recent simulations [25, 26] with atomistic potentials, whose greater complexity requires higher strain rates.

\section{E. Dissipative Stresses and Plasticity}

The large value of the hardening modulus and the multiplicative relation between it and flow stress, suggest that strain hardening is related to dissipation by plastic rearrangements rather than stored entropy. To quantify the rate of plastic deformation $R_{P}$, we examine changes in the Lennard-Jones bonds between monomers over small strain intervals $\delta \epsilon=0.005$. At the start of the interval, all bonds shorter than $r_{c}=1.5 \sigma$ are identified. Then the fraction $\delta f$ of these bonds whose length changes by more than $20 \%$ during the interval is evaluated. This threshold is large enough to exclude changes due to elastic deformations. Tests also show that the value of $\delta \epsilon=0.005$ is small enough that a given atom is unlikely to undergo multiple, independent bond rearrangements in any interval. To eliminate activated rearrangements associated with equilibrium aging, the rate of plasticity during deformation was monitored at $T=0$.

Figure 11(a) shows the rate of plasticity $R_{P} \equiv \delta f / \delta \epsilon$ as a function of $|g|$ during uniaxial compression of fully entangled $(N=350)$ and short $(N=4)$ chains with $k_{\text {bend }}=0.75$. The two chain lengths lead to very different curves, but for both cases $R_{P}$ is directly proportional to the dissipative component of the stress. To illustrate this we also plot $\sigma_{z}^{Q} / \sigma^{*}$ where $\sigma^{*}$ is the constant of proportionality relating $R_{P}$ and $\sigma_{z}^{Q}$. Note that even the rapid fluctuations with $|g|$ in the two quantities are correlated. These fluctuations are greatly reduced in the total stress, which can not be made to correlate as well with $R_{P}$. The $N=4$ chains exhibit nearly perfect-plastic behavior for $|g|>1$, showing that the correlation is not directly related to strain hardening. The fact that $\sigma^{*}$ is nearly the same for short chains that flow at a constant $\tau_{\text {flow }}$ and entangled chains that show significant strain hardening at larger strains is clear evidence for the close connection between the flow stress and strain hardening.

In Ref. [17] we showed that $R_{P}$ and $\sigma_{z}$ were also correlated for uniaxial compression of chains with $k_{\text {bend }}=0$ and $1.5 u_{0}$. Figure 12 shows that this connection extends to plane-strain compression. In all cases studied, $R_{P}$ tracks both the mean $\sigma_{z}^{Q}$ and local fluctuations. Moreover, the normalization constants have nearly the same value within our numerical uncertainties. Best fits for all $N, k_{\text {bend }}$ and strain states range between 0.98 and $1.1 u_{0} / a^{3}$. Since $R_{P}$ is the rate of rearrangements per LJ bond, $\sigma^{*}$ should correspond to the density of LJ bonds $\rho_{L J}$ times the energy dissipated per bond. Each atom has on average about 13 LJ neighbors and each bond is shared by two atoms, so $\rho_{L J} \sim 6.5 \rho$. Thus the energy dissipated per bond $\sigma^{*} / \rho_{L J}$ is about a quarter of the binding energy $\left(0.68 u_{0}\right)$. Note that this value would change slightly with the threshold used to define a bond rearrangement and other factors in the definition of $R_{P}$, but the result that $\sigma^{*}$ is similar for all systems with the same $r_{c}$ is more robust. Increasing $r_{c}$ increases the binding energy and also $\sigma^{*}$.

The same $\sigma^{*}$ are obtained when the strain rate is reduced to $10^{-5} / \tau_{L J}$, but the magnitude of the fluctuations increases slightly. Different behavior appears when the strain rate is increased to $10^{-3} / \tau_{L J}$. Fluctuations are much smaller since there is insufficient time for stress equilibration. There is also a decrease in $R_{P}$, while $\sigma_{z}^{Q}$ increases. This implies that there are fewer plastic rearrangements involving larger dissipation, presumably because the system does not have time to minimize the energy.

\section{SUMMARY AND CONCLUSIONS}

Extensive simulations of strain hardening were performed for polymer glasses with a wide range of entanglement densities, chain lengths and temperatures. As in experiments, we find that the calculated stress-strain curves of entangled chains can be fit to expressions derived from entropic network models (Eq. 6). These models normally treat the flow stress as an independent parameter that is determined by entirely separate mechanisms. However, our simulations [8] and experiments [47] show that $\tau_{\text {flow }}$ and $G_{R}$ are correlated, and that both drop linearly to zero as $T$ rises to $T_{g}$. This suggests that $\tau_{\text {flow }}$ enters multiplicatively rather than additively, and motivated a simple modification of the eight-chain model that describes the full temperature dependence of stress-strain curves. While this model may prove useful for extrapolating experimental data at one temperature to all others, the fit parameters do not appear to have physical significance. For example, values of $N_{e}$ are different for uniaxial and plane strain deformation. Another difficulty is that the model implies a relation between the two nonzero stress components in plane strain compression that is not satisfied by the data. We are not aware of experimental studies of the transverse stress $\sigma_{y}$, but it would be interesting to see if the same inconsistency could be observed experimentally.

Separate study of the energetic and thermal components of the stress provided insight into the failures of network models. The thermal component of the stress $\sigma^{Q}$ scales nearly linearly with $|g|$ for all systems. Even when $h$ is as large as 0.5 , the Langevin contribution to strain hardening (Eq. 6) is not evident in $\sigma^{Q}$. Instead the rapid rise in $\sigma$ at large $|g|$ is associated with an increase in the energetic component of stress. This rise 
is the dominant factor in fits of $N_{e}$ to the eight-chain model. Since the energetic contributions scale in different ways for uniaxial and plane strain, fit values of $N_{e}$ are different for the two strain states. Existing experiments have not examined energetic and thermal components of the stress separately in the strain hardening regime under isothermal conditions, but in principle deformation calorimetry experiments could do so. Our results suggest that a study of trends with entanglement density would be particularly useful.

Analysis of chain conformations reveals the origin of the energetic stress. As strain increases, chains are pulled taut over longer segments. When the length of straight segments reaches $N_{e}$, entanglements limit further straightening. Additional strain leads to a rapid increase in the tension in covalent bonds and the energetic component of stress. Straightening on a scale of order $N_{e}$ corresponds to large $h$. Thus even though different strain states lead to different fit values of $N_{e}$ in the eight-chain model, both fit values tend to follow trends in the true $N_{e}$. Modern microscopic theories of rubber elasticity [64] incorporate intra- and inter-chain energetic effects due to chain stretching and orientation, respectively. Analytic studies based on this approach [65] may be able to capture the changes in stress and chain conformation observed in our simulations.

Our simulations reproduce the shape recovery observed in experiments when strongly deformed, well-entangled glasses are unloaded and heated slightly above $T_{g}$ [28]. This relaxation is often invoked as evidence for the entropic stresses predicted by network models. As expected from this picture we find a strong correlation between relaxation of strain and the decay of strain-induced orientational order. However, we show that the stress associated with shape recovery is only of order $\rho_{e} k_{B} T$ and thus much too small to account for strain hardening. This stress was determined by measuring the shear stress in deformed samples after rapid heating, and by identifying the shear stress needed to strain an undeformed sample at the rate observed in shape recovery. The latter method could also be applied in experiments.

Limited orientation and shape recovery were observed for unentangled chains even though entropic models assume that there is no network to impose chain orientation in such systems. Significant strain hardening was also found for these unentangled chains. The stress and orientation follow results for highly entangled chains at small $|g|$ and saturate at large $|g|$. The onset of satura- tion moves to larger $|g|$ as $N$ increases. As suggested by recent theoretical work [29] and observed in our recent simulations [17], the stress is directly related to effective stretches describing the microscopic chain orientation $\lambda_{i}^{c}$ rather than the macroscopic stretches $\lambda_{i}$. Plots of stress against $g\left(\bar{\lambda}^{c}\right)$ collapse data for unentangled and highly entangled chains onto a single curve. Small deviations from this collapse are observed when the energetic contribution to the stress is large, i.e. when $N_{e}$ is small and strain is large.

For both entangled and unentangled chains the thermal contribution to the stress is directly proportional to the rate of bond rearrangements. Both the gradual trends and rapid fluctuations in the two quantities track each other. The proportionality constant is nearly independent of $N_{e}$ and chain length. The latter result helps to explain the connection between $\tau_{\text {flow }}$ and $G_{R}$ since short chains shear at a constant stress near $\tau_{\text {flow }}$. There has been great interest recently in plasticity in model atomic glasses [66, 67, 68]. It would be interesting to check whether the direct correlation between dissipative stress and bond breaking/reformation holds in such systems [69].

One of the intriguing questions raised by our results is why the thermal contribution to the stress always rises nearly linearly with $|g|$. The stress has the functional form expected for the entropy of Gaussian chains even in the limit $T \rightarrow 0$ where entropic contributions to the stress must vanish. One possibility is that entropy enters indirectly. As $|g|$ increases, the number of conformations available to the chains is reduced. The growing constraint on conformations may naturally lead to an increase in the number of local bond rearrangements that scales with entropy. This would explain the linear increase in the rate of plasticity with $|g|$, and the corresponding increase in $\sigma^{Q}$.

\section{ACKNOWLEDGEMENTS}

This material is based upon work supported by the National Science Foundation under Grant No. DMR0454947. We thank G. S. Grest for providing equilibrated states and K. S. Schweizer and D. J. Read for useful discussions. Simulations were performed with the LAMMPS software package (http://lammps.sandia.gov).
[1] R. N. Haward and G. Thackray, Proc. Roy. Soc. Lond. 302, 453 (1968).

[2] E. M. Arruda and M. C. Boyce, Int. J. Plast. 9, 697 (1993).

[3] E. M. Arruda, M. C. Boyce, and R. Jayachandran, Mech. Mat. 19, 193 (1995).

[4] M. C. Boyce, E. M. Arruda, and R. Jayachandran,
Polym. Eng. Sci. 34, 716 (1994).

[5] H. Ping and W. Demin, Act. Mech. Solida Sinica 12, 36 (1999).

[6] E. J. Kramer, J. Polym. Sci. Part B - Polym. Phys. 43, 3369 (2005).

[7] H. G. H. van Melick, L. E. Govaert, and H. E. H. Meijer, Polymer 44, 2493 (2003). 
[8] R. S. Hoy and M. O. Robbins, J. Polym. Sci. Part B Polymer Phys. 44, 3487 (2006).

[9] C. G'sell and A. Souahi, in Deformation Yield and Fracture of Polymers (The Insititute of Materials, London, 1994), p. 29.

[10] F. M. Capaldi, M. C. Boyce, and G. C. Rutledge, Phys. Rev. Lett 89, 175505 (2002).

[11] L. S. Loo, R. E. Cohen, and K. K. Gleason, Science 288, 5463 (2000).

[12] K. Chen and K. S. Schweizer, Europhys. Lett. 79, 26006 (2007).

[13] O. A. Hasan and M. C. Boyce, Polymer 34, 5085 (1993).

[14] D. Rittel, Mech. Mat. 31, 131 (1999).

[15] Z. Li and J. Lambros, Int. J. Solids Struct. 38, 3549 (2001).

[16] C. Chui and M. C. Boyce, Macromolecules 32, 3795 (1999).

[17] R. S. Hoy and M. O. Robbins, Phys. Rev. Lett. 99, 117801 (2007).

[18] J. Li, T. Mulder, B. Vorselaars, A. V. Lyulin, and M. A. J. Michels, Macromolecules 39, 7774 (2006).

[19] D. Brown and J. H. R. Clarke, 24, 2075 (1991).

[20] J. I. Mckechnie, R. N. Haward, D. Brown, and J. H. R. Clarke, Macromolecules 26, 198 (1993).

[21] P. H. Mott, A. S. Argon, and U. W. Suter, Philosoph. Mag. A 67, 931 (1993).

[22] S. S. Jang and W. H. Jo, J. Chem. Phys. 110 (1999).

[23] F. M. Capaldi, M. C. Boyce, and G. C. Rutledge, Polymer 45, 1391 (2004).

[24] A. Fortunelli, C. Geloni, and A. Lazzeri, J. Chem. Phys. 121, 4941 (2004).

[25] A. V. Lyulin, N. K. Balabaev, M. A. Mazo, and M. A. J. Michels, Macromolecules 37, 8785 (2004).

[26] A. V. Lyulin, B. Vorselaars, M. A. Mazo, N. K. Balabaev, and M. A. J. Michels, Europhys. Lett, 71, 618 (2005).

[27] K. Kremer and G. S. Grest, J. Chem. Phys. 92, 5057 (1990).

[28] R. N. Haward and R. J. Young, eds., The Physics of Glassy Polymers, 2nd edition (Chapman and Hall (London), 1997).

[29] This idea was suggested by K. S. Schweizer and forms the basis of a microscopic theory of strain hardening 65]. The underlying physics relates to the theoretical prediction that the bulk modulus of strained melts and rubbers increases with chain stretching 64].

[30] J. Rottler and M. O. Robbins, Phys. Rev. E 68, 011801 (2003).

[31] R. Auhl, R. Everarers, G. S. Grest, K. Kremer, and S. J. Plimpton, J. Chem. Phys. 119, 12718 (2003).

[32] R. Everaers, S. K. Sukumaran, G. S. Grest, C. Svaneborg, A. Sivasubramanian, and K. Kremer, Science 303, 823 (2004).

[33] D. Frenkel and B. Smit, Understanding Molecular Simulations, 2nd edition (Academic Press (San Diego), 2002).

[34] T. Schneider and E. Stoll, Phys. Rev. B 17, 1302 (1978).

[35] J. Rottler and M. O. Robbins, Phys. Rev. E 68, 011507 (2003).

[36] J. Rottler and M. O. Robbins, Phys. Rev. E 64, 051801 (2001).

[37] Q. Zhou and R. G. Larson, Macromolecules 38, 5761 (2005).

[38] R. S. Hoy and M. O. Robbins, Phys. Rev. E 72, 061802 (2005).

[39] E. J. Kramer, Adv. Polym. Sci 52/53, 1 (1983).
[40] R. N. Haward, Macromolecules 26, 5860 (1993).

[41] L. Yang, D. J. Srolovitz, and A. F. Yee, J, Chem. Phys 107, 4396 (1997).

[42] J. Rottler and M. O. Robbins, Phys. Rev. Lett. 95, 225504 (2005).

[43] A. D. Mulliken and M. C. Boyce, Int J. Solids and Structures 43, 1331 (2006).

[44] M. C. Boyce, D. M. Parks, and A. S. Argon, Mech. Mat. 7, 15 (1988).

[45] The model is less successful at predicting results for uniaxial tension; see e.g. Ref. 70].

[46] J. Richeton, S. Ahzi, K. S. Vecchio, F. C. Jiang, and A. Makradi, Int. J. Solids Stuct. 44, 7938 (2007).

[47] R. B. Dupaix and M. C. Boyce, Polymer 46, 4827 (2005).

[48] M. Wendlandt, T. A. Tervoort, and U. W. Suter, Polymer 46, 11786 (2005).

[49] H. G. H. van Melick, L. E. Govaert, and H. E. H. Meijer, Polymer 44, 3579 (2003).

[50] F. Varnik, L. Bocquet, and J.-L. Barrat, J. Chem. Phys. 120, 2788 (2004).

[51] G. W. Adams and R. J. Ferris, J. Polym.. Sci. Part B Polym. Phys. 26, 433 (1988).

[52] O. B. Salamatina, G. W. H. Höhne, S. N. Rudnev, and E. F. Oleinik, Thermochimica Acta 247, 1 (1994).

[53] E. F. Oleinik, S. N. Rudnev, O. B. Salamatina, S. V. Shenogin, M. I. Kotelyanskii, T. V. Paramzina, and S. I. Nazarenko, e-Polymers 029 (2006).

[54] E. Oleynik, Prog. Coll. Polym. Sci. 80, 140 (1989).

[55] S. V. Shenogin, G. W. H. Höhne, and E. F. Oleinik, Thermochim. Acta 391, 13 (2002).

[56] Note that the $N_{e}=71$ chains appear to deform slightly superaffinely (by $<0.5 \%$ ) at moderate strains. This is a finite size effect. It arises from the use of the formula $R_{a f f}(n)=\lambda_{\text {chain }} R_{0}(n)$, which assumes the averages over chains of the Cartesian components of $R_{0}^{2}(n)$ are isotropic. In a finite system there are always small deviations from anisotropy. Correcting for them does not change other features in the plot.

[57] Of course $R(n)$ is an average over all points on the chain. One might imagine that there are localized entanglement points that deform more affinely or that there is a distribution of entanglement separations as seen in simulations [71]. While this might preserve the idea of an affinely deformed network, the discrepancy from the eight-chain model would remain.

[58] M. Warner and S. F. Edwards, J. Phys. A Math. Gen. 8, 1649 (1978).

[59] D. J. Read, unpublished.

[60] M. Warren and J. Rottler, Phys. Rev. E 76, 031802 (2007).

[61] A. S. Argon, Philos. Mag. 28, 39 (1973).

[62] M. Doi and S. F. Edwards, The Theory of Polymer Dynamics (Clarendon Press (Oxford), 1986).

[63] Because the magnitude of the stress difference is smaller, the fluctuations between curves appear greater than in Fig. 8 .

[64] F. T. Oyerokun and K. S. Schweizer, J. Chem. Phys. 120, 9359 (2004).

[65] K. Chen and K. S. Schweizer, unpublished.

[66] C. E. Maloney and A. Lemaitre, Phys. Rev.. E 74, 016118 (2006).

[67] A. Tanguy, F. Leonforte, and J. L. Barrat, Eur. Phys. J. E 20, 355 (2006).

[68] N. P. Bailey, J. Schiotz, A. Lemaitre, and K. W. Jacob- 
sen, Phys. Rev. Lett. 98, 095501 (2007).

[69] Unpublished work by B.-Q. Luan and M. O. Robbins shows that it does in friction of amorphous solids.

[70] W. H. Han, F. Horcay, and G. B. McKenna, Math. Mech.
Solids 4, 139 (1999).

[71] C. Tzoumanekas and D. N. Theodorou, Macromolecules 39, 4592 (2006). 


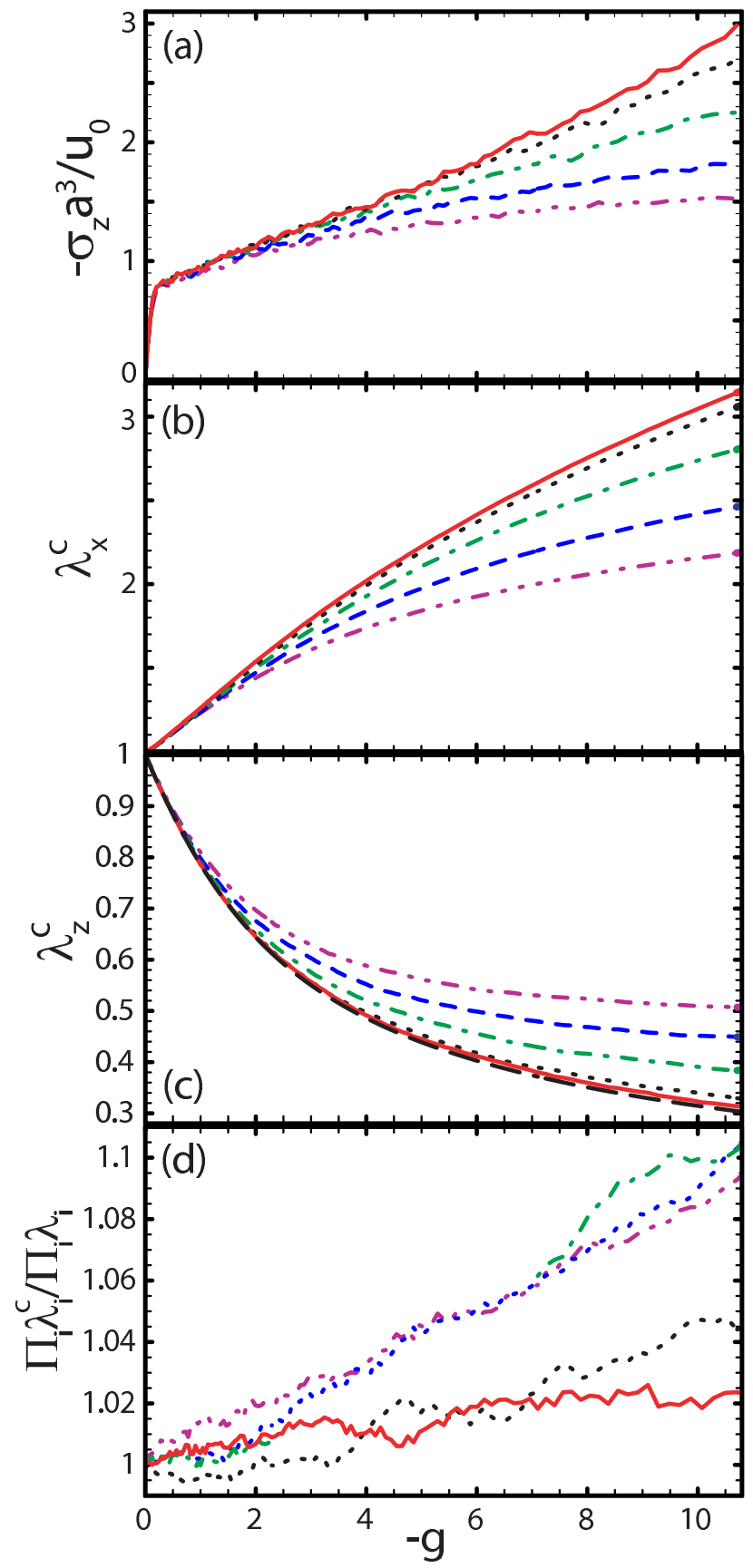

FIG. 7: (Color online) Dependence of (a) stress and (b,c) microscopic chain orientation on $|g|$ for flexible chains under plane strain compression at $T=0.2 u_{0} / k_{B}$ with $\dot{\epsilon}=$ $-10^{-5} / \tau_{L J}$. Panel (d) shows the ratio of changes in chain volume to macroscopic volume. The chains have lengths $N=500$ (solid lines), $N=107$ (dotted lines), $N=36$ (dashdotted lines), $N=18$ (dashed lines), and $N=12$ (dashdot-dotted lines). The lower dashed line in (c) shows the macroscopic stretch $\lambda_{z}$. 

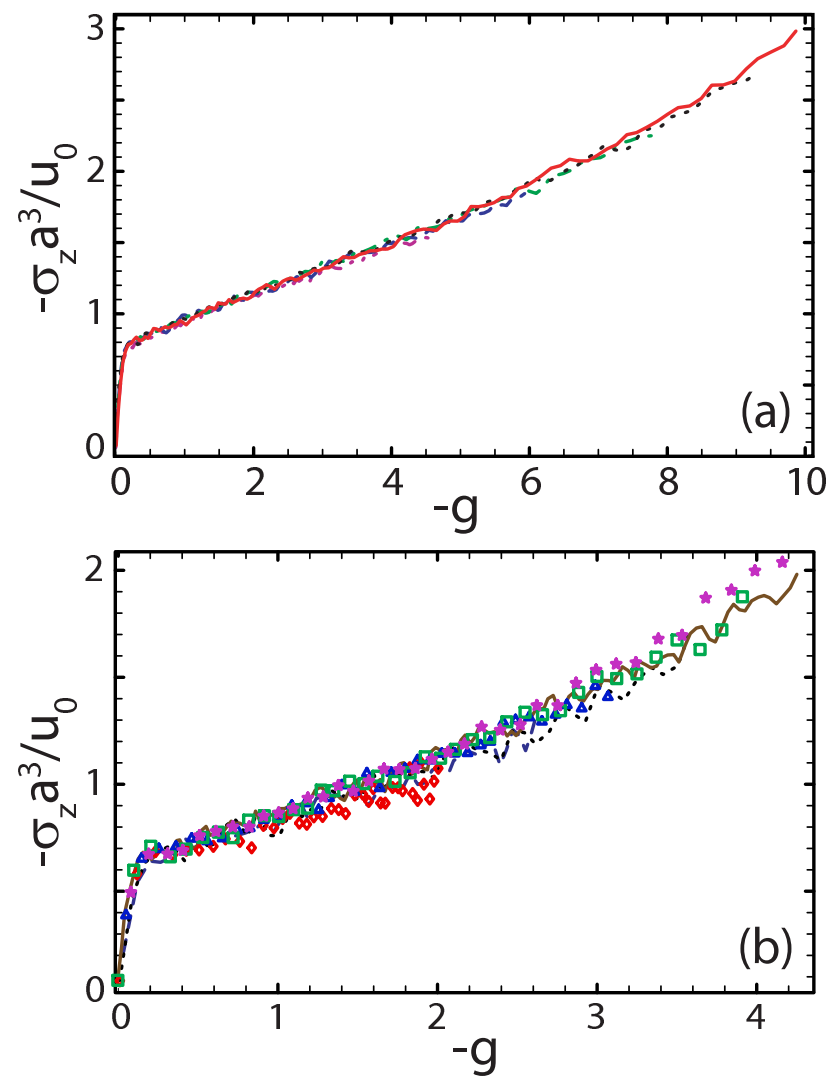

FIG. 8: (Color online) Compressive stress as a function of microscopic orientation function $g_{\text {eff }} \equiv\left(\lambda_{z}^{c}\right)^{2}-\left(\lambda_{x}^{c}\right)^{2}$ at $T=0.2 u_{0} k_{B}$ with $\dot{\epsilon}=-10^{-5} / \tau_{L J}$. (a) Plane strain compression of flexible chains with lengths $N=500$ (solid line), 107 (dotted line), 36 (dash-dotted line), 18 (dashed line), or 12 (dot-dash-dot line). (b) Uniaxial compression of semiflexible chains $\left(N_{e}=39\right)$ with $N=350(\star), 175(-), 70$ (squares), 40 $(\cdots), 25(\triangle), 16(---)$ and $10(\diamond)$. 


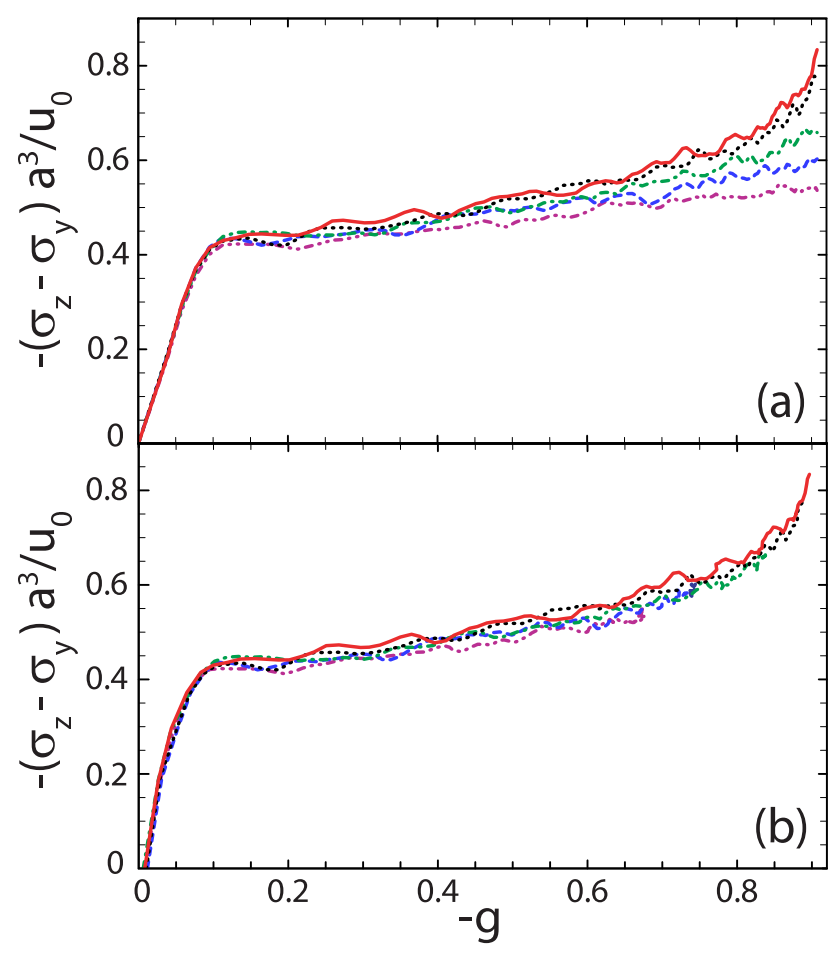

FIG. 9: (Color online) Stress difference $\sigma_{z}-\sigma_{y}$ as a function of (a) the macroscopic $g$ and (b) the microscopic orientation function $g_{\text {eff }} \equiv\left(\lambda_{z}^{c}\right)^{2}-\left(\lambda_{y}^{c}\right)^{2}$ at $T=0.2 u_{0} k_{B}$ with $\dot{\epsilon}=-10^{-5} / \tau_{L J}$. Chains have length $N=500$ (solid line), 107 (dotted line), 36 (dash-dotted line), 18 (dashed line), or 12 (dot-dash-dot line). 


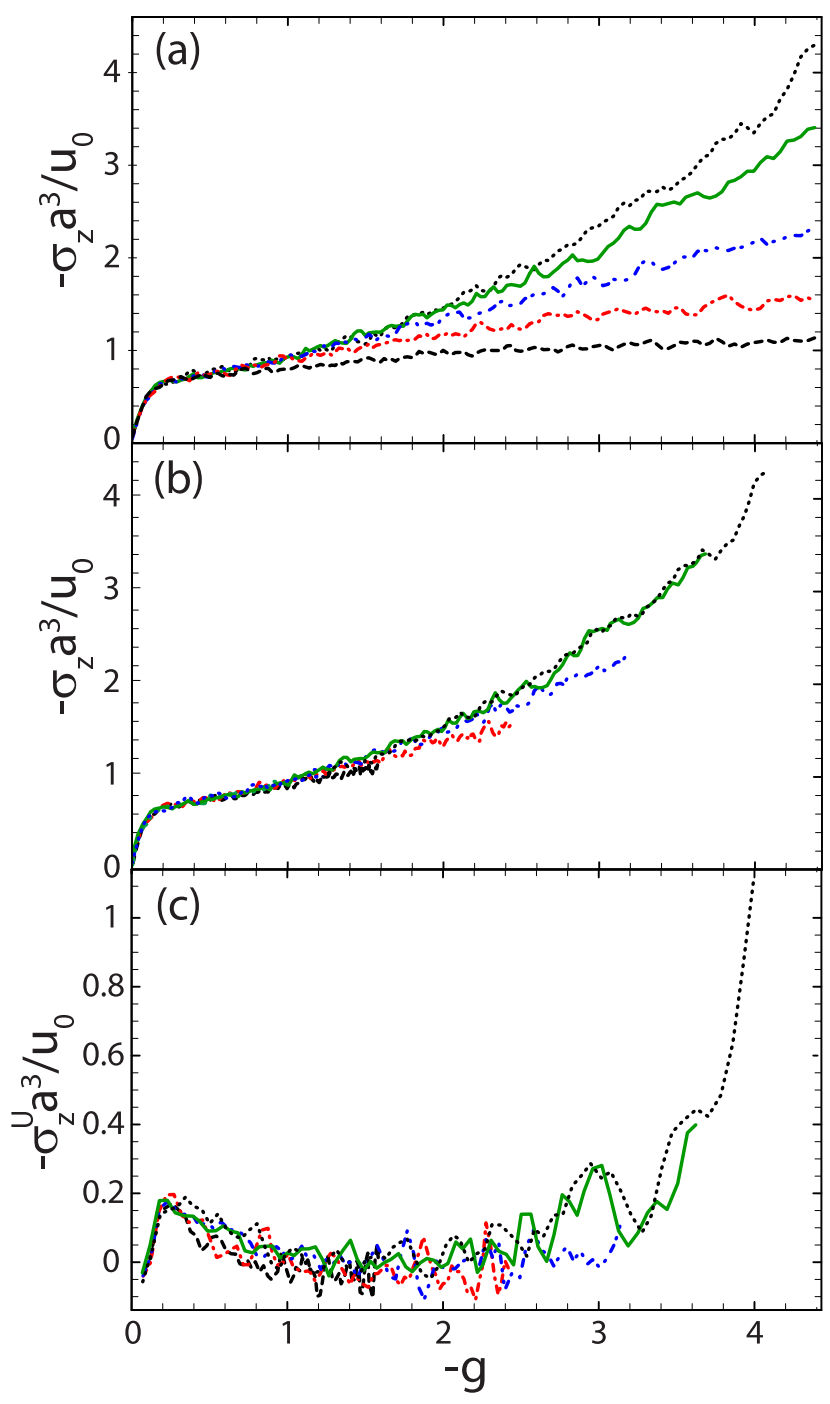

FIG. 10: (Color online) (a) Stress as a function of $g$ during uniaxial compression of $k_{\text {bend }}=2.0 u_{0}$ chains with $N=350$ (dotted), 88 (solid), 44 (dash-dot-dotted), 22 (dash-dotted) and 11 (dashed) at $T=0.2 u_{0} / k_{B}$ and $\dot{\epsilon}=-10^{-5} / \tau_{L J}$. (b) Stress replotted against $g_{\text {eff }}$. (c) Energetic stress $\sigma_{z}^{U}$ plotted against $g_{\text {eff }}$. 


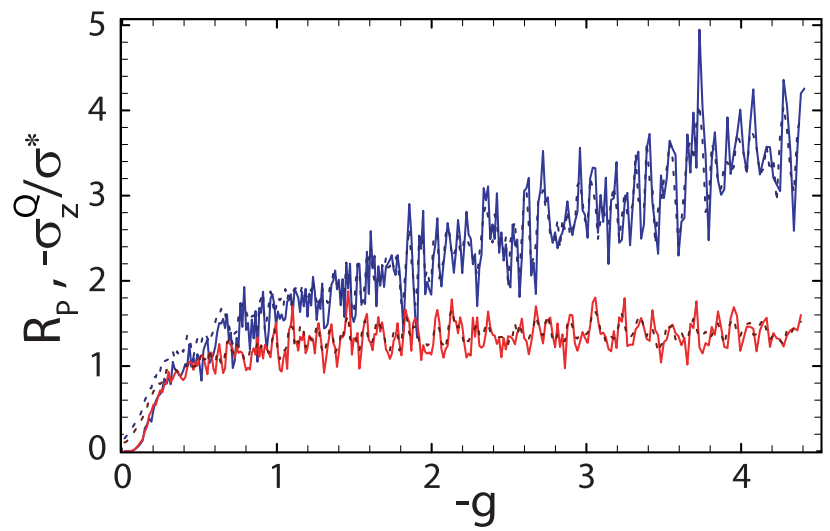

FIG. 11: (Color online) Rate of plastic deformation $R_{P}$ (solid lines) and normalized dissipative stress (dashed lines) for uniaxial compression of $k_{\text {bend }}=0.75 u_{0}$ chains with $N=350$ (upper curves) and $N=4$ (lower curves). Here $T=0$, $\dot{\epsilon}=-10^{-4} / \tau_{L J}$ and $\sigma^{*}=1.02 u_{0} / a^{3}$ for $N=350$ and $1.1 u_{0} / a^{3}$ for $N=4$.

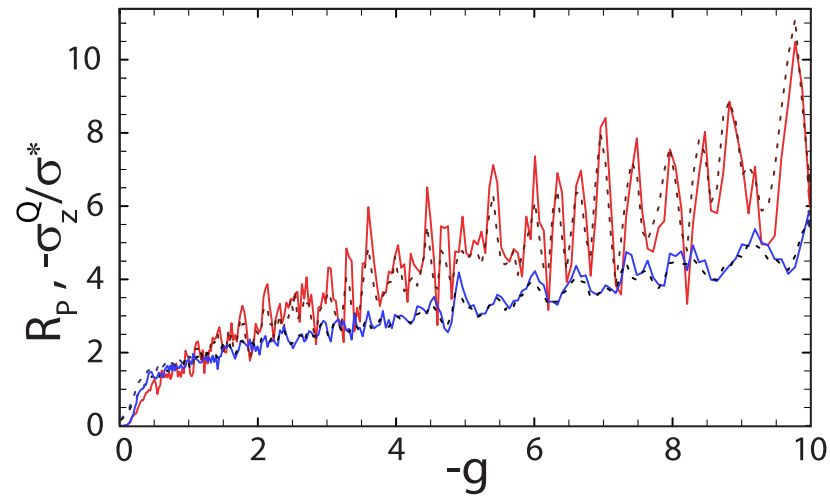

FIG. 12: (Color online) Rate of plasticity (solid lines) and dissipative stress (dashed lines) for plane strain compression of flexible ( $\left.k_{\text {bend }}=0\right) N=500$ chains (lower curves) and semiflexible $\left(k_{\text {bend }}=1.5 u_{0}\right) N=350$ chains (upper curves). Here $T=0, \dot{\epsilon}=-10^{-4} / \tau_{L J}$ and $\sigma^{*}=1.05 u_{0} / a^{3}$ for $k_{\text {bend }}=$ $1.5 u_{0}$ and $0.98 u_{0} / a^{3}$ for $k_{\text {bend }}=0 u_{0}$. 